\title{
Macroeconomic Stabilization Policies in Intrinsically Unstable Macroeconomies*
}

\author{
Carl Chiarella, Peter Flaschel, Carsten Köper, Christian Proaño, and Willi \\ Semmler
}

\begin{abstract}
Many monetary and fiscal policy measures have aimed at mitigating the effects of the financial market meltdown that started in the U.S. subprime sector in 2008 and has subsequently spread world wide as a great recession. Slowly some recovery appears to be on the horizon, yet it is worthwhile exploring the fragility and potentially destabilizing feedbacks of advanced macroeconomies in the context of a framework that builds on the ideas of Keynes and Tobin. This framework stresses the fragilities and destabilizing feedback mechanisms that are potential features of all major markets- - those for goods, labor, and financial assets. We use a Tobin macroeconomic portfolio approach and the interaction of heterogeneous agents on the financial market to characterize the potential for financial market instability. Though the study of the latter has been undertaken in many partial models, we focus here on the interconnectedness of all three markets. Furthermore, we study what potential labor market, fiscal and monetary policies can have in stabilizing unstable macroeconomies. In order to study this problem we introduce, besides money, long term bonds and equity into the asset market. We in particular propose a countercyclical monetary policy that sells assets in the boom and purchases them in recessions. Modern stability analysis is brought to bear to demonstrate the stabilizing effects of the suggested policies. The policies suggested here could help the Fed in its search for an appropriate exit strategy after its massive intervention in the financial market.
\end{abstract}

* The authors acknowledge the comments and suggestions from the audience at the conference in honour of Professor Giancarlo Gandolfo. The usual caveat applies. 


\section{Introduction}

The impact of financial market crises on the real side of the economy has long been studied. ${ }^{1}$ Yet, the current financial crisis is less well understood. It seems to be neither a financial crisis triggered by a currency run, nor by the bursting of a technology bubble, but rather a crisis originating in the financial market in one of the most advanced countries of the world economy, the US. It appears to have resulted from two driving forces: macroeconomic changes (low interest rates, high liquidity, easy credit, and external imbalances) and the use of new financial innovations which substantially contributed to increasing leverage and driving up asset prices.

The crisis is also to some extent a crisis in economic theory. Since about the mid 1970s, economists have come to accept the dynamic stochastic general equilibrium (DSGE) framework as the one in which to carry out macroeconomic analysis. The earlier Keynesian (and other variants) have been dismissed as being "old fashioned" and/or no longer relevant. This approach survives in what today may be called the non-market clearing disequilibrium paradigm or tradition (see Chiarella, Flaschel and Franke (2005)); it stresses in particular the main macroeconomic (stabilising/destabilising) feedback mechanisms and their interconnectedness, the lack of which in the DSGE paradigm makes that framework problematic. The difference in viewpoints may be seen as a continuation of the discussion from the 1930s as to whether the origin of business cycles is due to some endogenous mechanism that can constrain an economic system that has the potential to become locally unstable; or, whether it is generated by a system that is locally stable but continually perturbed by external shocks. The fact that when faced with such a deep crisis policy makers essentially went back to the old Keynesian policy prescriptions is, in the view of the authors, an indictment of the standard theory and casts some doubt on its relevance, even though it has become so dominant.

The financial crisis, starting in the US subprime sector, has spread world wide to become a great recession. A hyperactive monetary and fiscal policy since the end of 2007 has attempted to prevent a further financial meltdown in the advanced economies. Whilst some observers maintain that a slow recovery appears to be on the horizon and that the standard economic paradigm has redeemed itself, it is still worth exploring the fragility and potentially destabilizing feedbacks of advanced macroeconomies in the context of the non-market clearing macroeconomic paradigm. This is particularly so since fragilities and destabilizing feedbacks seem to be potential features of all markets (the product market, the labor market, and

\footnotetext{
${ }^{1}$ An important framework for studying the history of financial crises can be found in Minsky (1982).
} 
the financial market), and the period we have just lived through could easily occur again.

In this paper we will focus in particular on the financial market. We use a Tobin type of macroeconomic portfolio approach, coupled with the interaction of heterogeneous agents on the financial market, to characterize the potential for financial market instability. Though the study of the latter has been undertaken in many partial models, we focus here on the interconnectedness of all three markets. Furthermore, we study the potential for labor market and monetary policies to stabilise unstable macroeconomies. In a companion paper, Asada, Chiarella, Flaschel, Mouakil, Proaño and Semmler (2011), we focus on fiscal policy measures that can stabilise the economy. Amongst a range of stabilizing policies we in particular propose a countercyclical monetary policy that sells assets in the boom and purchases them in recessions. Modern stability analysis of dynamical systems is brought to bear to demonstrate the stabilizing effects of the suggested policies.

This paper builds on work of Asada, Chiarella, Flaschel, Mouakil, Proaño and Semmler (2010), Asada et al. (2011) by using the modelling framework of those papers to design a macrodynamic framework for labor market and monetary policies that allows in general for large swings in financial and real economic activity. It builds on models of the dynamic interaction of the labor market, the product market and the financial market with risky assets. We revive the framework of a macroeconomic portfolio approach that Tobin $(1969,1980,1982)$ had suggested, but also build on recent work on the interaction of heterogeneous agents in the financial market. ${ }^{2}$ We allow for heterogeneity in share and goods price expectations and study the financial, nominal and real cumulative feedback chains that may give rise to the potential of an unstable economy. Whilst we would characterise our approach as a disequilibrium one, we do connect to traditional Keynesian business cycle analysis as proposed by writers such as Tobin and Minsky. An important addition to the related papers by Asada et al. $(2010,2011)$, is the incorporation of long term bonds on the asset market in order to capture some essential features of the financial sector. We will show that, on the one hand, the insights gained in Asada et al. (2010), concerning safe and risky assets can be preserved and meaningfully extended while, on the other hand, the role of monetary policy is reformulated in ways that make it effective. The enhancement of the financial markets by one further risky asset does not restructure their working in a fundamental way, yet it will allow the redesigned monetary policy to overcome its somewhat unrealistic working (through open market operations on the stock market).

\footnotetext{
${ }^{2}$ Recent work stressing the interaction of fundamentalist and behavioural traders in financial markets and their role in creating bubbles and crashes, includes Abreu and Brunnermeier (2003) and Chiarella, Dieci and He (2009).
} 
The model consists of households, divided into worker households and asset holder households; and firms who produce and invest; and fiscal and monetary policy authorities who provide the economy public services within the limits of their budget constraints. An important module is a wage price sector which relies upon both a dynamic wage Phillips curve and a dynamic price Phillips curve, both of which are affected by a medium run expected inflation rate that adjusts in an adaptive fashion. The capital markets equilibrate the demand for the financial assets, money, equity and short term bonds, and in this paper long-term bonds, and are based upon Tobin's (1969) framework as embellished by Franke and Semmler (1999). The framework overall stresses the causal downward nexus from financial market dynamics to real economic activity and stresses very much the feedback mechanisms between the various sectors of the economy. In this respect, it differs markedly from the DSGE approach. The main difference between the model presented in Asada et al. (2011) and the current paper is the use of long term bonds rather than short term bonds as one of the assets of the model. This change may at first seem minor but in fact is quite significant as it allows us to capture some essential features of monetary policy.

The paper is organized as follows. Section 2 motivates long-term bonds and incorporates them formally into the model structure. The intensive form of the model and its steady state are introduced in Section 3. The comparative statics and the dynamics are studied in Section 4 and Section 5 explores a range of policies that can bring about macroeconomic stability. Section 6 proposes a new form of monetary policy that is not only concerned with interest rates, but also with the countercyclical selling and buying of assets, a policy that the US Fed in fact has undertaken and which is, in spirit, close to ideas proposed by Minsky (1982). Section 7 concludes the paper.

\section{Adding long-term bonds}

In the basic KMG-Tobin model of Asada et al. (2011) money, short term bonds, and equities comprise the set of financial assets. These assets were chosen in that paper because the primary goal was to make clear the way in which the financial part of the economy with equilibrated markets, when embedded with the real part of the economy, brings about an inherent disequilibrium. One good reason to extend this restricted set of financial assets in order to include long term bonds, is that they compete more directly with the equity markets. They are also more comparable with respect to the time horizon of investment. In the following, we shall refer to equity $(E)$, bonds $\left(B, B^{l}\right)$ and money $(M)$ as the financial assets in the economy. 
Sargent (1987, p.12) notes with respect to the bond $(B)$, that it is a variablecoupon bond that is issued by the government. The bond is essentially a savings deposit, with changes in the interest rate altering the coupon, but leaving the dollar value of bonds outstanding unchanged. This characterization implies that such bonds are part of the money supply that is just $M_{2}$ (which is the broad monetary aggregate consisting of demand deposits, savings and other time deposits). The question arises whether we should use a financial structure where the government can issue money (in the sense of the money supply measure $M_{2}$ ). Some assets issued by the government exhibit a high degree of liquidity (due to their short maturity horizon), but the bulk of government expenditure is not financed in this way, and in particular not at a constant price of issue, since this would not only interfere with the objectives of the central bank, but the bonds issued in the past would also be an ideal object for rising liquidity preference (hoarding), due to the positive yield this asset provides, in particular when it is considered as a perfect substitute for equities as in Sargent (1987). As shown in Asada et al. (2010, 2011) under mild assumptions on the portfolio demand functions for the financial assets consisting of money, equity and short term bonds (around the steady state) monetary policy is completely ineffective, since it only influences the cash management process between money and bonds, but does not reach the equity market and thus the financial structure of firms.

Since in macroeconomic analysis one has to economize on the use of financial instruments in order to remain tractable we will use the theoretically polar case to fix-price bonds, namely perpetuities in place of short-term bonds to finance the government deficit. Short term bonds will be present implicitly, since we will use the monetary aggregate $M_{2}=M+B$ in the following analysis and they will only be considered explicitly in Section 6 on monetary policy. Perpetuities are the other typical bond configuration used in continuous time macrodynamics. Note that, if we were to merely replace $B$ by long-term bonds $B^{l}$ with a variable price $p_{b}$ and with the actual rate of return $1 / p_{b}+\hat{p}_{b}$, we would lose an equation for the determination of the interest rate. In this way we are able to add long-term bonds to the model without increasing its complexity, since taxes are treated net of interest transfers as in Sargent (1987, p.16) and Rødseth (2000, p.170).

On the basis of the foregoing discussion we are dealing with the set of financial assets: money $M_{2}$, long term bonds $B^{l}$ and equities $E$. The long term bonds are perpetuities valued at any point in time with price $p_{b}$ and they pay out one unit of money in every period of time, displaying an expected rate of return

$$
r_{b}^{e}=1 / p_{b}+\pi_{b}
$$


which is the sum of the inverted bond price (the interest rate), and the expected capital gain (the expected growth rate in long term bond prices $\pi_{b}$ ). ${ }^{3}$ The actual composition of $M_{2}$ will only matter when monetary policy is considered explicitly in Section 6. As the general structure is very similar to that of Asada et al. (2011) we only give detailed explanation where the changes impact on the structural equations.

\subsection{Private households}

The worker households are not affected at all as they are assumed to consume all their income and save nothing, and so are not involved in asset markets. However asset holder households will experience some changes. The equations for their income, consumption and savings become (note that $p$ is the price of output $)^{4}$

$$
\begin{aligned}
C_{c} & =\left(1-s_{c}\right)\left[r_{k}^{e} K+\left(B^{l}+i B\right) / p-T_{c}\right], \\
S_{c} & =s_{c}\left[r_{k}^{e} K+\left(B^{l}+i B\right) / p-T_{c}\right]=\left(\dot{M}_{2}+p_{b} \dot{B}^{l}+p_{e} \dot{E}\right) / p, \\
W_{c} & =\left(M_{2}+p_{b} B^{l}+p_{e} E\right) / p, \quad W_{c}^{n}=p W_{c} .
\end{aligned}
$$

The sources of income consist of dividend payments of firms to asset holders $\left(r_{k}^{e} K\right)$ and real interest payments for the bond holdings $\left(B^{l}+i B\right) / p$. The income is diminished by a $\operatorname{tax} T_{c}$. Thus consumption and saving are given by the saving propensity $s_{c}$ of asset holders according to equations (2) and the first equation of (3).

The saving of asset holders enters their financial wealth. The desired nominal amounts of the financial assets are determined by demand functions that depend on the rates of return of the assets and wealth according to the demand functions ${ }^{5,6}$

\footnotetext{
${ }^{3} \mathrm{We}$ assume that perpetuities $B^{l}$ (issued by the government) have an expected rate of return $1 / p_{b}+\pi_{b}$, and equities $E$ (issued by firms) an expected rate of return $r_{e}^{e}=\left(p Y-w L^{d}-\delta K\right) /\left(p_{e} E\right)+$ $\pi_{e}$, where $p Y-w L^{d}-\delta K$ is the dividend payments of firms, $p_{e}$ is the price of equities and $\pi_{e}$ is the expected growth rate in equity prices.

${ }^{4}$ Even though we have hidden the short term bonds in the expression for $M_{2}$, interest on these in the form of $i B$ still needs to be taken into account in the following discussion. The reader should also keep in mind that since the long term bonds pay out one unit of money per unit time the interest payment on them (in nominal terms) appears simply as $B^{l}$ in (2) and (3).

${ }^{5} \mathrm{We}$ make the assumption that the nominal rate of interest has no impact on the asset demands; which may be justified around the steady state of the economy (assuming that it only has an impact on the cash management process within the money supply $M_{2}$, but not on the risky assets).

${ }^{6}$ Note that $r_{e}^{e}$, the expected rate of return on equity, is defined at equation (24) below as $r_{e}^{e}=$ $\left(Y^{e}-\delta K-\omega L^{d}\right) / K=r_{k}^{e} / q+\pi_{e}$ where $r_{k}^{e}$ is the expected return on capital.
} 


$$
\begin{aligned}
M_{2}^{d} & =f_{m}\left(r_{b}^{e}, r_{e}^{e}\right) W_{c}^{n}, \\
p_{b} B^{l d} & =f_{b^{l}}\left(r_{b}^{e}, r_{e}^{e}\right) W_{c}^{n}, \\
p_{e} E^{d} & =f_{e}\left(r_{b}^{e}, r_{e}^{e}\right) W_{c}^{n}, \\
W_{c}^{n} & =M_{2}^{d}+p_{b} B^{l d}+p_{e} E^{d} .
\end{aligned}
$$

\subsection{Government}

The taxation of asset holders by the government changes and hence the government budget equations must be adjusted to

$$
\begin{aligned}
T & =\tau_{w} \omega L^{d}+T_{c}, \\
T_{c} & =t_{c} K+\left(B^{l}+i B\right) / p, \text { with } t_{c}=\text { const. } \\
G & =g K, \quad g=\text { const. } \\
S_{g} & =T-\left(B^{l}+i B\right) / p-G, \\
\hat{M}_{2} & =\mu, \\
\dot{M}_{2}+p_{b} \dot{B}^{l} & =p G+B^{l}+i B-p T .
\end{aligned}
$$

In equation (10) real tax levied on asset holders consists of a fixed proportion on total capital $t_{c} K$ and of interest payments on the stock of bonds. ${ }^{7}$ This is a standard "trick" to avoid interest payments in the consumption function of asset holders and, as we will see, plays a role in the government budget constraint. It also has the effect of making the model's dynamics independent of $i B$, the interest payments on short term bonds, as it avoids the feedback of interest payments on asset holders' consumption and their savings decision. The saving of the government is then given by the income $T=\tau_{w} \omega L^{d}+T_{c}$ minus the government purchases for interest payments and provision of government services. From all this we can derive the government budget equation as stated in equation (14), which state that all purchases minus income are financed by the issue of money or long term bonds. ${ }^{8}$

\footnotetext{
${ }^{7}$ The reader should be aware of that even though equities and long-term bonds are taxed differently, it is implicit in the assumed portfolio demand functions that there is no arbitrage opportunity between these two financial assets.

${ }^{8}$ The relationship between short-term and long-term interest rates is implicit in the assumed portfolio demand functions, which are based on expected rates of returns on long-term bonds and equities.
} 


\subsection{Asset markets}

As in the modelling of the asset holders, the demands for financial assets depend crucially on the rates of return on long term bonds and equities. Thus, in order to reach an equilibrium at every point in time on money, bond and equity markets, the rates of return have to adjust to their equilibrium values instantaneously. Naturally the demand functions of the asset holders in the capital markets fulfill the adding up constraints

$$
f_{m}+f_{b^{l}}+f_{e}=1
$$

and

$$
\frac{\partial f_{m}}{\partial z}+\frac{\partial f_{b^{l}}}{\partial z}+\frac{\partial f_{e}}{\partial z}=0, \quad \text { for } \quad z \in\left\{r_{b}^{e}, r_{e}^{e}\right\}
$$

We also assume that the gross substitution property of the demand function is met.

\section{Intensive form of the model and its steady state}

For notational simplicity we use the symbol $m$ in place of $m_{2}=m+b$ until we return to an explicit treatment of the composition of the variable $m_{2}$ in Section 6. In order to express the model in capital intensive form we use the results of Asada et al. (2011). However in addition we need to derive explicitly the law of motion for the intensive form of long term bonds $b^{l}=\frac{B^{l}}{p K} \cdot{ }^{9}$

Solving equation (14) with respect to $\dot{B}^{l}$ yields

$$
\dot{B}^{l}=\frac{1}{p_{b}}\left(p\left(g-t_{c}\right) K-\tau_{w} w L^{d}-\dot{M}_{2}\right)
$$

and substituting this relation into $\dot{b}^{l}=\frac{\partial\left(\frac{B^{l}}{p K}\right)}{\partial t}=\frac{\dot{B}^{l}}{p K}-b^{l}(\hat{p}+I / K)$ we obtain

$$
\dot{b}^{l}=\frac{1}{p_{b}}\left(g-t_{c}-\tau_{w} \omega l^{d}-\mu m\right)-b^{l}(\hat{p}+I / K) .
$$

Then substituting the expression for $\hat{p}^{10}$ into (18) we find (here $\pi^{c}$ is the inflation climate variable, the formation of which is discussed at equation (39) below)

$$
\dot{b}^{l}=\frac{1}{p_{b}}\left(g-t_{c}-\tau_{w} \omega l^{d}-\mu m\right)-b^{l}\left(\kappa\left[\beta_{p}(u-\bar{u})+\kappa_{p} \beta_{w}(e-\bar{e})\right]+\pi^{c}+i(\cdot)\right) .
$$

\footnotetext{
${ }^{9}$ Note that $b^{l}$ is the number of long-term bonds per unit value of the capital stock.

${ }^{10}$ See (37) below for the equation for $\hat{p}$.
} 
With these preliminary considerations the entire model can be set up. First we lay out the statically endogenous variables needed later in the differential equations:-

$$
\begin{aligned}
y & =\left(1+\alpha_{n^{d}}\left(n+\beta_{n}\right)\right) y^{e}-\beta_{n} v \\
l^{d} & =y / x \\
c & =\left(1-\tau_{w}\right) \omega l^{d}+\left(1-s_{c}\right)\left(y^{e}-\delta-\omega l^{d}-t_{c}\right) \\
i(\cdot) & =i_{q}(q-1)+i_{u}(u-\bar{u})+n \\
y^{d} & =c+i(\cdot)+\delta+g \\
r_{e}^{e} & =\frac{r_{k}^{e}}{q}+\pi_{e} \\
r_{b}^{e} & =\frac{1}{p_{b}}+\pi_{b} \\
\pi_{e} & =\alpha_{\pi_{e}} \pi_{e c}+\left(1-\alpha_{\pi_{e}}\right) \pi_{e f} \\
\pi_{b} & =\alpha_{\pi_{b}} \pi_{b c}+\left(1-\alpha_{\pi_{b}}\right) \pi_{b f} \\
e & =l^{d} / l \\
u & =y / y^{p} \\
r_{k}^{e} & =y^{e}-\delta-\omega l^{d} .
\end{aligned}
$$

In the above $q$ is Tobin's $q$ and is defined as the value of equity over the value of capital stock, that is $q=p_{e} E /(p K)$. Tobin's $q$ and the price of long term bonds $p_{b}$ are responsible for the equilibrium on the financial markets for money, long term bonds and equities at every point in time. The intensive form of the demand functions reads

$$
\begin{aligned}
m^{d} & =f_{m}\left(r_{b}^{e}, r_{e}^{e}\right)\left(m+p_{b} b^{l}+q\right) \\
p_{b} b^{l d} & =f_{b^{l}}\left(r_{b}^{e}, r_{e}^{e}\right)\left(m+p_{b} b^{l}+q\right) \\
q & =f_{e}\left(r_{b}^{e}, r_{e}^{e}\right)\left(m+p_{b} b^{l}+q\right) .
\end{aligned}
$$

Thus the solution of the two equilibrium conditions

$$
\begin{gathered}
f_{m}\left(\frac{1}{p_{b}}+\pi_{b}, \frac{r_{k}^{e}}{q}+\pi_{e}\right)\left(m+p_{b} b^{l}+q\right)-m=0, \\
f_{b^{l}}\left(\frac{1}{p_{b}}+\pi_{b}, \frac{r_{k}^{e}}{q}+\pi_{e}\right)\left(m+p_{b} b^{l}+q\right)-b=0,
\end{gathered}
$$

gives combinations of $q$ and $p_{b}$ that equilibrate the money market and the long-term bond market. ${ }^{11}$ The growth rates of wages and prices are given by the wage and

\footnotetext{
${ }^{11}$ A solution will automatically equilibrate the equity market because of Walras' law.
} 
price Phillips curves

$$
\begin{aligned}
& \hat{w}=\kappa\left(\beta_{w}(e-\bar{e})+\kappa_{w} \beta_{p}(u-\bar{u})\right)+\pi^{c}, \\
& \hat{p}=\kappa\left(\beta_{p}(u-\bar{u})+\kappa_{p} \beta_{w}(e-\bar{e})\right)+\pi^{c}
\end{aligned}
$$

from which one can derive the growth rate of real wages (see equation (38) below).

The differential equations of the model can be expressed as

$$
\begin{aligned}
\hat{\omega}= & \kappa\left[\left(1-\kappa_{p}\right) \beta_{w}(e-\bar{e})+\left(\kappa_{w}-1\right) \beta_{p}(u-\bar{u})\right] \\
\dot{\pi}^{c} & =\alpha \beta_{\pi^{c}} \kappa\left[\beta_{p}(u-\bar{u})+\kappa_{p} \beta_{w}(e-\bar{e})\right]+(1-\alpha) \beta_{\pi^{c}}\left(\mu-n-\pi^{c}\right), \\
\hat{l}= & n-i(\cdot)=-i_{q}(q-1)-i_{u}(u-\bar{u}) \\
\dot{y}^{e}= & \beta_{y^{e}}\left(y^{d}-y^{e}\right)+(n-i(\cdot)) y^{e} \\
\dot{v}= & y-y^{d}-i(\cdot) v \\
\dot{\pi}_{b f}^{c}= & \beta_{\pi_{b f}}\left(\bar{\eta}_{b}-\pi_{b f}\right) \\
\dot{\pi}_{b c}= & \beta_{\pi_{b c}}\left(\hat{p}_{b}-\pi_{b c}\right) \\
\dot{\pi}_{e f}= & \beta_{\pi_{e f}}\left(\bar{\eta}_{e}-\pi_{e f}\right) \\
\dot{\pi}_{e c}= & \beta_{\pi_{e c}}\left(\hat{p}_{e}-\pi_{e c}\right) \\
\dot{b}^{l}= & \frac{1}{p_{b}}\left(g-t_{c}-\tau_{w} \omega l^{d}-\mu m\right) \\
& -b\left(\kappa\left[\beta_{p}(u-\bar{u})+\kappa_{p} \beta_{w}(e-\bar{e})\right]+\pi^{c}+i(\cdot)\right) \\
\dot{m}= & m \mu-m\left(\kappa\left[\beta_{p}(u-\bar{u})+\kappa_{p} \beta_{w}(e-\bar{e})\right]+\pi^{c}+i(\cdot)\right) .
\end{aligned}
$$

Note that the statically endogeneous quantities defined in equations (19) (30) are required to form the right hand sides of the dynamical system (38) - (48). form:- ${ }^{12}$

We can state the following result about the steady state of the intensive

\section{Theorem 1 Assume that}

1. the saving propensity of the asset holders $s_{c}$ is sufficiently large;

2. the government runs a primary deficit;

3. the long term expectations for equity price inflation of the fundamentalists equals the steady state inflation rate of the prices of goods;

\footnotetext{
${ }^{12}$ From this section onwards we prove a number of propositions and theorems. The propositions usually deal with the stability of the dynamical system under consideration whilst the theorems deal with policy prescriptions. Generally we give the proofs of the propositions and, in the interests of keeping the paper within reasonable bounds, omit the proofs of the theorems as they are quite similar. The only exception is Theorem 8 .
} 
4. setting $\bar{\phi}=\frac{g-t_{c}-\tau_{w} \omega l^{d}}{g-t_{c}-\tau_{w} \omega l^{d}+\mu}$, the demand functions for financial assets are such that

$$
\begin{aligned}
& \lim _{r_{b}^{e} \rightarrow 0}\left(f_{m}\left(r_{b}^{e}, r_{k}^{e}+\pi_{e}\right)+f_{b}\left(r_{b}^{e}, r_{k}^{e}+\pi_{e}\right)\right)<\bar{\phi} \\
& \lim _{r_{b}^{e} \rightarrow \infty}\left(f_{m}\left(r_{b}^{e}, r_{k}^{e}+\pi_{e}\right)+f_{b}\left(r_{b}^{e}, r_{k}^{e}+\pi_{e}\right)\right)>\bar{\phi}
\end{aligned}
$$

then the dynamical system (38) to (48) displays a unique interior steady state with $\omega^{o}, l^{o}, y^{e o}, v^{o}, b^{o}, m^{o}>0$.

Proof: See Köper (2003).

\section{Financial markets: Comparative statics}

We have shown that under the conditions stated in Theorem 1 there exists a unique steady state. Without loss of generality we specify the demand functions for financial assets in a neighborhood of the steady state by (the $\alpha_{x 0}, \alpha_{x 1}, \alpha_{x 2}$ for $x \in$ $\{m, b, e\}$ are all $>0$.)

$$
\begin{array}{r}
f_{m}\left(r_{b}^{e}, r_{e}^{e}\right)=\alpha_{m 0}-\alpha_{m 1} r_{b}^{e}-\alpha_{m 2}\left(\frac{r_{k}^{e}}{q}+\pi_{e}\right) \\
f_{b^{l}}\left(r_{b}^{e}, r_{e}^{e}\right)=\alpha_{b^{l} 0}+\alpha_{b^{l} 1} r_{b}^{e}-\alpha_{b^{l} 2}\left(\frac{r_{k}^{e}}{q}+\pi_{e}\right), \\
f_{e}\left(r_{b}^{e}, r_{e}^{e}\right)=\alpha_{e 0}-\alpha_{e 1} r_{b}^{e}+\alpha_{e 2}\left(\frac{r_{k}^{e}}{q}+\pi_{e}\right) .
\end{array}
$$

Denoting the excess demand for cash balances and long term bonds by $F_{1}$ and $F_{2}$ respectively and using (25) we have

$$
\begin{aligned}
& F_{1}\left(p_{b}, q ; \pi_{b}, r_{k}^{e}, \pi_{e}, b^{l}, m\right) \\
& \quad=\left[\alpha_{m 0}-\alpha_{m 1}\left(\frac{1}{p_{b}}+\pi_{b}\right)-\alpha_{m 2}\left(\frac{r_{k}^{e}}{q}+\pi_{e}\right)\right]\left(m+p_{b} b^{l}+q\right)-m, \\
& F_{2}\left(p_{b}, q ; \pi_{b}, r_{k}^{e}, \pi_{e}, b^{l}, m\right) \\
& \quad=\left[\alpha_{b^{l} 0}+\alpha_{b^{l} 1}\left(\frac{1}{p_{b}}+\pi_{b}\right)-\alpha_{b^{l}}\left(\frac{r_{k}^{e}}{q}+\pi_{e}\right)\right]\left(m+p_{b} b^{l}+q\right)-p_{b} b^{l} .
\end{aligned}
$$

At equilibrium $F_{1}=0$ and $F_{2}=0$ and applying the implicit function theorem

$$
\left(\begin{array}{c}
\frac{\partial p_{b}}{\partial m} \\
\frac{\partial q}{\partial m}
\end{array}\right)=-\left(\begin{array}{ll}
\frac{\partial F_{1}}{\partial p_{b}} & \frac{\partial F_{1}}{\partial q} \\
\frac{\partial F_{2}}{\partial p_{b}} & \frac{\partial F_{2}}{\partial q}
\end{array}\right)^{-1}\left(\begin{array}{c}
\frac{\partial F_{1}}{\partial m} \\
\frac{\partial F_{2}}{\partial m}
\end{array}\right):=J^{(2)^{-1}}\left(\begin{array}{c}
\frac{\partial F_{1}}{\partial m} \\
\frac{\partial F_{2}}{\partial m}
\end{array}\right)
$$


We compute

$$
\begin{aligned}
\operatorname{det} J^{(2)}= & \left|\begin{array}{ll}
\frac{\partial F_{1}}{\partial p_{b}} & \frac{\partial F_{1}}{\partial q} \\
\frac{\partial F_{2}}{\partial p_{b}} & \frac{\partial F_{2}}{\partial q}
\end{array}\right| \\
= & \left(\alpha_{m 1} \alpha_{b^{l} 2}+\alpha_{b^{l} 1} \alpha_{m 2}\right) \frac{1}{p_{b}^{2}} \frac{r_{k}^{e}}{q^{2}}\left(m+p_{b} b^{l}+q\right)^{2}+\alpha_{m 1} \frac{1}{p_{b}^{2}} p_{b} b^{l}+\alpha_{b^{l} 2} \frac{r_{k}^{e}}{q^{2}} m b^{l} \\
& +\alpha_{b^{l} 1} \frac{1}{p_{b}^{2}} m+\alpha_{m 2} \frac{r_{k}^{e}}{q^{2}}(m+q) b^{l}+\frac{m b^{l}}{m+p_{b} b^{l}+q}
\end{aligned}
$$

From the latter we can easily conclude that $\operatorname{det} J^{(2)}>0$ because all terms of the sum are positive. In the following we make extensive use of the positive sign of the determinant. To check the qualitative influence of the exogenous variables on the price for long term bonds we only have to evaluate the sign of the term

$$
\frac{1}{\operatorname{det} J^{(2)}}\left(\frac{\partial F_{1}}{\partial q} \frac{\partial F_{2}}{\partial z}-\frac{\partial F_{2}}{\partial q} \frac{\partial F_{1}}{\partial z}\right) \quad \text { for } \quad z \in\left\{\pi_{e}, \pi_{b}, r_{k}^{e}, m, b^{l}\right\}
$$

and for qualitative influence on Tobin's $q$, that of

$$
\frac{1}{\operatorname{det} J^{(2)}}\left(\frac{\partial F_{2}}{\partial p_{b}} \frac{\partial F_{1}}{\partial z}-\frac{\partial F_{1}}{\partial p_{b}} \frac{\partial F_{2}}{\partial z}\right) \quad \text { for } \quad z \in\left\{\pi_{e}, \pi_{b}, r_{k}^{e}, m, b^{l}\right\} .
$$

We evaluate the comparative statics of the dynamics around the steady state.

The effect of changes in money on the price of bonds:

$$
\begin{aligned}
\frac{\partial p_{b}}{\partial m} & =\frac{1}{\operatorname{det} J^{(2)}}\left(\frac{\partial F_{1}}{\partial q} \frac{\partial F_{2}}{\partial m}-\frac{\partial F_{2}}{\partial q} \frac{\partial F_{1}}{\partial m}\right) \\
& =\frac{1}{\operatorname{det} J^{(2)}}\left(\alpha_{m 2} \frac{r_{k}^{e}}{q^{2}} p_{b} b^{l}+\alpha_{b^{l} 2} \frac{r_{k}^{e}}{q^{2}}\left(p_{b} b^{l}+q\right)+\frac{p_{b} b^{l}}{m+p_{b} b^{l}+q}\right)
\end{aligned}
$$

which is positive since there are no negative terms in the sum. Thus, a rise (fall) in money stock leads to a rising (falling) $p_{b}$.

The effect of changes in long term bonds on the bond price:

$$
\begin{aligned}
\frac{\partial p_{b}}{\partial b^{l}} & =\frac{1}{\operatorname{det} J^{(2)}}\left(\frac{\partial F_{1}}{\partial q} \frac{\partial F_{2}}{\partial b^{l}}-\frac{\partial F_{2}}{\partial q} \frac{\partial F_{1}}{\partial b^{l}}\right) \\
& =\frac{p_{b}}{\operatorname{det} J^{(2)}}\left(-\alpha_{m 2} \frac{r_{k}^{e}}{q^{2}}(m+q)-\alpha_{b^{l}} \frac{r_{k}^{e}}{q^{2}} m-\frac{m}{m+p_{b} b^{l}+q}\right)<0
\end{aligned}
$$

so that a rise in the supply of long term bonds lowers the price of the bonds. 
The dependence of the bond price on expected bond price inflation:

$$
\begin{aligned}
& \frac{\partial p_{b}}{\partial \pi_{b}}=\frac{1}{\operatorname{det} J^{(2)}}\left(\frac{\partial F_{1}}{\partial q} \frac{\partial F_{2}}{\partial \pi_{b}}-\frac{\partial F_{2}}{\partial q} \frac{\partial F_{1}}{\partial \pi_{b}}\right) \\
& =\frac{1}{\operatorname{det} J^{(2)}}\left(\alpha_{m 2} \frac{r_{k}^{e}}{q^{2}}\left(m+p_{b} b^{l}+q\right)+\frac{m}{m+p_{b} b^{l}+q}\right)\left(\alpha_{b^{l} 1}\left(m+p_{b} b^{l}+q\right)\right) \\
& -\frac{1}{\operatorname{det} J^{(2)}}\left(\alpha_{b^{l} 2} \frac{r_{k}^{e}}{q^{2}}\left(m+p_{b} b^{l}+q\right)+\frac{p_{b} b^{l}}{m+p_{b} b^{l}+q}\right)\left(-\alpha_{m 1}\left(m+p_{b} b^{l}+q\right)\right)>0
\end{aligned}
$$

which has a positive impact.

The dependence of the bond prices on expected equity price inflation:

$$
\begin{aligned}
\frac{\partial p_{b}}{\partial \pi_{e}} & =\frac{1}{\operatorname{det} J^{(2)}}\left(\frac{\partial F_{1}}{\partial q} \frac{\partial F_{2}}{\partial \pi_{e}}-\frac{\partial F_{2}}{\partial q} \frac{\partial F_{1}}{\partial \pi_{e}}\right) \\
& =\frac{1}{\operatorname{det} J^{(2)}}\left(\left(-\alpha_{b^{l} 2} m\right)-\left(-\alpha_{m 2} p_{b} b^{l}\right)\right)=?
\end{aligned}
$$

The ? indicates that we cannot say anything definite about this impact. It depends on the exact sizes of the parameters of the system.

Dependence of bond prices on the expected rate of profit:

$$
\begin{aligned}
\frac{\partial p_{b}}{\partial r_{k}^{e}} & =\frac{1}{\operatorname{det} J^{(2)}}\left(\frac{\partial F_{1}}{\partial q} \frac{\partial F_{2}}{\partial r_{k}^{e}}-\frac{\partial F_{2}}{\partial q} \frac{\partial F_{1}}{\partial r_{k}^{e}}\right) \\
& =\frac{1}{\operatorname{det} J^{(2)}}\left(\left(-\alpha_{b^{l} 2} / q m\right)-\left(-\alpha_{m 2} / q p_{b} b^{l}\right)\right)=?
\end{aligned}
$$

Again we cannot determine the sign of this term.

The effect of money supply on Tobin's $q$ :

$$
\begin{aligned}
\frac{\partial q}{\partial m} & =\frac{1}{\operatorname{det} J^{(2)}}\left(\frac{\partial F_{2}}{\partial p_{b}} \frac{\partial F_{1}}{\partial m}-\frac{\partial F_{1}}{\partial p_{b}} \frac{\partial F_{2}}{\partial m}\right) \\
& =\frac{1}{\operatorname{det} J^{(2)}}\left(\left(\alpha_{b^{l} 1}-\alpha_{m 1}\right) \frac{1}{p_{b}^{2}} p_{b} b^{l}+\alpha_{b^{l}} \frac{1}{p_{b}^{2}} q+\frac{b^{l} q}{m+p_{b} b^{l}+q}\right)
\end{aligned}
$$

which is positive because the gross substitution property gives $\left(\alpha_{b^{l} 1}-\alpha_{m 1}\right)>0$ and so we can conclude that $\frac{\partial q}{\partial m}>0$. 
The effect of long term bond supply on Tobin's q:

$$
\begin{aligned}
\frac{\partial q}{\partial b^{l}} & =\frac{1}{\operatorname{det} J^{(2)}}\left(\frac{\partial F_{2}}{\partial p_{b}} \frac{\partial F_{1}}{\partial b^{l}}-\frac{\partial F_{1}}{\partial p_{b}} \frac{\partial F_{2}}{\partial b^{l}}\right) \\
& =\frac{1}{\operatorname{det} J^{(2)}}\left(-\alpha_{b^{l} 1} \frac{1}{p_{b}} m+\alpha_{m 1} \frac{1}{p_{b}}(m+q)\right)
\end{aligned}
$$

thus

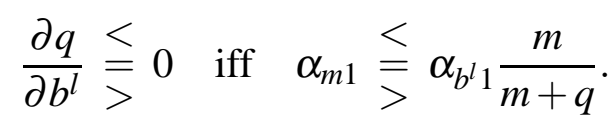

The dependence of Tobin's $q$ on expected bond price inflation:

$$
\begin{aligned}
\frac{\partial q}{\partial \pi_{b}} & =\frac{1}{\operatorname{det} J^{(2)}}\left(\frac{\partial F_{2}}{\partial p_{b}} \frac{\partial F_{1}}{\partial \pi_{b}}-\frac{\partial F_{1}}{\partial p_{b}} \frac{\partial F_{2}}{\partial \pi_{b}}\right) \\
& =\frac{1}{\operatorname{det} J^{(2)}}\left(\alpha_{m 1}(m+q) b^{l}-\alpha_{b^{l} 1} m b^{l}\right) .
\end{aligned}
$$

Again the sign of this quantity is not definite and so depends on the particular parameter values.

The dependence of Tobin's $q$ on expected equity price inflation:

$$
\begin{aligned}
& \frac{\partial q}{\partial \pi_{e}}=\frac{1}{\operatorname{det} J^{(2)}}\left(\frac{\partial F_{2}}{\partial p_{b}} \frac{\partial F_{1}}{\partial \pi_{e}}-\frac{\partial F_{1}}{\partial p_{b}} \frac{\partial F_{2}}{\partial \pi_{e}}\right)= \\
& \frac{1}{\operatorname{det} J^{(2)}}\left(-\alpha_{b^{l} 1} \frac{1}{p_{b}^{2}}\left(m+p_{b} b^{l}+q\right)+\left(\frac{p_{b} b^{l}}{m+p_{b} b^{l}+q}-1\right) b^{l}\right)\left(-\alpha_{m 2}\left(m+p_{b} b^{l}+q\right)\right) \\
& -\frac{1}{\operatorname{det} J^{(2)}}\left(\alpha_{m 1} \frac{1}{p_{b}^{2}}\left(m+p_{b} b^{l}+q\right)+\frac{m}{m+p_{b} b^{l}+q} b^{l}\right)\left(-\alpha_{b^{l} 2}\left(m+p_{b} b^{l}+q\right)\right)>0 .
\end{aligned}
$$

The dependence of Tobin's $q$ on the expected rate of profit:

$$
\begin{aligned}
& \frac{\partial q}{\partial r_{k}^{e}}=\frac{1}{\operatorname{det} J^{(2)}}\left(\frac{\partial F_{2}}{\partial p_{b}} \frac{\partial F_{1}}{\partial r_{k}^{e}}-\frac{\partial F_{1}}{\partial p_{b}} \frac{\partial F_{2}}{\partial r_{k}^{e}}\right) \\
& =\frac{1}{q \operatorname{det} J^{(2)}}\left(\left(\alpha_{b^{l} 1} \alpha_{m 2}+\alpha_{m 1} \alpha_{b^{l} 2}\right)\left(m+p_{b} b^{l}+q\right)^{2}+\alpha_{m 2} b^{l}(m+q)+\alpha_{b^{l}{ }^{2}} m b^{l}\right)>0 .
\end{aligned}
$$

In this case the impact is unambiguous. 
The following proposition turns out to be of use in subsequent analysis:-

Proposition 1 Tobin's $q$ in the steady state satisfies the relation

$$
\frac{\partial q}{\partial m}>\frac{\partial q}{\partial\left(p_{b} b^{l}\right)}
$$

Proof: We readily calculate from the comparative static results provided above that there holds

$$
\left(\alpha_{b^{l} 1}-\alpha_{m 1}\right) \frac{1}{p_{b}^{2}} p_{b} b^{l}+\alpha_{b^{l} 1} \frac{1}{p_{b}^{2}} q+\frac{b^{l} q}{m+p_{b} b^{l}+q}>\left(\alpha_{m 1}-\alpha_{b^{l} 1}\right) \frac{1}{p_{b}^{2}} m+\alpha_{m 1} \frac{1}{p_{b}^{2}} q
$$

from which it follows that

$$
\left(\alpha_{b^{l} 1}-\alpha_{m 1}\right) \frac{1}{p_{b}^{2}}\left(m+p_{b} b^{l}+q\right)+\frac{b^{l} q}{m+p_{b} b^{l}+q}>0 .
$$

We know that $\left(\alpha_{b^{l} 1}-\alpha_{m 1}\right)>0$ and all other variables are positive in the steady state. $^{13}$

\section{Policies for the stability of balanced growth}

In this section we shall develop some policies about the stability of the dynamical system driving the macroeconomy. Our proof strategy will be to prove results for lower dimensional systems and gradually extend these by adding one dimension at a time until we obtain the full dynamics. Our approach relies very much on the continuity of the eigenvalue structure with respect to small changes in the underlying parameters. ${ }^{14}$ Theorem 2 below relies on the existence of an independent fiscal authority. It is true that such a concept may not yet be realised but we remind the reader that it is not so long ago that the concept of independent monetary authority (which is now the norm in most advanced economies) was equally far-fetched. For further discussion on this we refer the reader to Asada et al. (2011).

First we prove the following proposition and theorem for the 3D subsystem:-

Proposition 2 Assume that the values of $\omega, v, \pi^{c}, \pi_{e c}, \pi_{e f}, \pi_{b c}$, and $\pi_{b f}$ are at their steady state values and that the parameters $\beta_{p}, \beta_{w}, \beta_{n}, \beta_{\pi^{c}}, \beta_{\pi_{e c}}, \beta_{\pi_{e f}}, \beta_{\pi_{b c}}$,

\footnotetext{
${ }^{13}$ The reader should note that $\left.\frac{\partial q}{\partial m}\right|_{m=p_{b}} b^{l}>0$, which is reminiscent of the old textbook literature on government expenditures comparing money, bond and tax financing. Here the condition states that monetary expansion via open market bond purchases increases the state of confidence in the investment function and is thus expansionary.

${ }^{14}$ This approach is outlined in Chiarella, Flaschel, Franke and Semmler (2006) in a model that contains some of the features of the present model.
} 
$\beta_{\pi_{b f}}=0$, so that $\omega, v, \pi^{c}, \pi_{e c}, \pi_{e f}, \pi_{b c}$, and $\pi_{b f}$ will remain at their steady state values. In addition assume that the conditions for the existence of a steady state in Theorem 1 are fulfilled. Then the 3D dynamical system

$$
\begin{aligned}
\dot{m} & =m \mu-m\left(\pi_{o}^{c}+i(\cdot)\right), \\
\dot{b}^{l} & =\frac{1}{p_{b}(\cdot)}\left(g-t_{c}-\tau_{w} \omega l^{d}-\mu m\right)-b^{l}\left(\pi_{o}^{c}+i(\cdot)\right), \\
\dot{y}^{e} & =\beta_{y^{e}}\left(y^{d}(\cdot)-y^{e}\right)+(n-i(\cdot)) y^{e},
\end{aligned}
$$

possesses a locally asymptotically stable steady state under the following conditions: $\beta_{y^{e}}$ is sufficiently large, $i_{u}$ is sufficiently small, and the parameter $\alpha_{e 1}$ is sufficiently small, and $\alpha_{m 2}, \alpha_{b^{l} 2}$ are sufficiently small (that is, iff the parameter $\alpha_{e 2}$ is also sufficiently small, implying that the impact of changing $r_{e}^{e}$ is relatively moderate).

Proof: See the appendix.

Theorem 2 Assume, in addition to the government expenditure rules given by the equations (9) to (11) the existence of an independent fiscal authority solely responsible for the control of business fluctuations (acting independently of the fiscal policy given in (9) to (11)) which implements the following two rules for its activity oriented expenditures and their funding:

$$
g^{u}=-g_{u}(u-\bar{u}) \text { and } t^{u}=g_{u}(u-\bar{u}) .
$$

The budget of this authority is always balanced and it is assumed - due to the present form of the model - that the taxes $t^{u}$ are paid by (or in the boom received by) asset holding households. The stability condition on $i_{u}$ is now extended to the consideration of the parameter $\left(i_{u}-g_{u}\right)$. Then: An anti-cyclical policy $g_{u}$ that is chosen in a sufficiently active way will enforce damped oscillations in the $3 D$ subdynamics if the savings rate $s_{c}$ of asset holders is sufficiently close to one and if stock markets are sufficiently tranquil (meaning that the parameters $\alpha_{e 1}, \alpha_{e 2}$ are sufficiently small and $\alpha_{m 1}$ is not too large).

The theorem tells us that an anti-cyclical fiscal policy that is chosen in a sufficiently active way will enforce damped oscillations in the 3D subdynamics if the savings rate of asset holders is sufficiently close to one and if the stock market readjustments are sufficiently inelastic (and $\alpha_{m 1}$ not too large). Note that neither the steady state nor the laws of motions are changed through this introduction of such a self-determined business cycle authority, if $s_{c}=1$ holds true, which we assume 
to be the case. We also assume that the parametric assumptions on the financial markets can be made if the Tobin taxes (considered below) are chosen sufficiently large.

Next we let real wages vary by allowing the adjustment speed $\beta_{p}$ to be positive, so that we are dealing with the 4D system

$$
\begin{aligned}
\dot{m} & =m \mu-m\left(\kappa \beta_{p}\left(\frac{y}{y^{p}}-\bar{u}\right)+\pi_{o}^{c}+i(\cdot)\right), \\
\dot{b}^{l} & =\frac{1}{p_{b}}\left(g-t_{c}-\tau_{w} \omega l^{d}-\mu m\right)-b^{l}\left(\kappa \beta_{p}\left(\frac{y}{y^{p}}-\bar{u}\right)+\pi_{o}^{c}+i(\cdot)\right), \\
\dot{y}^{e} & =\beta_{y^{e}}\left(y^{d}-y^{e}\right)+y^{e}(n-i(\cdot)), \\
\dot{\omega} & =\omega \kappa\left(\kappa_{w}-1\right) \beta_{p}\left(\frac{y}{y_{p}}-\bar{u}\right) .
\end{aligned}
$$

Proposition 3 Under the assumptions made in Theorem 1 and in Proposition 2, it follows that the system (54) possesses an asymptotically stable steady state if the parameter $\beta_{p}$, the adjustment of real wages with respect to the capacity utilization gap $(u-\bar{u})$, is sufficiently small.

Proof: See the appendix.

Note that the implication of this condition for the $4 \mathrm{D}$ subdynamics is also obtained under the assumption $\kappa_{w}=1$, so that workers and their representatives should always demand a full indexation of their nominal wages to the rate of price inflation. This implies:

Theorem 3 Assume in the dynamical system (54) that the cost-push term in the money wage adjustment rule is given by the current rate of price inflation (which is perfectly foreseen). Then the $4 D$ subdynamics exhibit damped oscillations around the given steady state position of the economy.

This type of income policy, in fact of a scala mobile type, thus implies stability instead of instability (as might be expected), since it simplifies considerably the real wage channel.

We next enlarge the system by allowing $\beta_{w}$ to take positive values, so we are dealing with a 5D system about which we can state:

Proposition 4 Assume that the assumptions made in Proposition 3 are satisfied, but in addition allow the parameter $\beta_{w}$ to take positive values. Then the resulting 
dynamical system

$$
\begin{aligned}
\dot{m} & =m \mu-m\left(\kappa\left[\beta_{p}\left(\frac{y}{y^{p}}-u\right)+\kappa_{p} \beta_{w}\left(\frac{y}{x l}-\bar{e}\right)\right]+\pi_{o}^{c}+i(\cdot)\right) \\
\dot{b}^{l} & =\frac{1}{p_{b}}\left(g-t_{c}-\tau_{w} \omega \frac{y}{x}-\mu m\right)-b^{l}\left(\kappa\left[\beta_{p}\left(\frac{y}{y^{p}}-\bar{u}\right)+\kappa_{p} \beta_{w}\left(\frac{y}{x l}-\bar{e}\right)\right]+\pi_{o}^{c}+i(\cdot)\right), \\
\dot{y}^{e} & =\beta_{y^{e}}\left[c+i(\cdot)+\operatorname{det} J+g-y^{e}\right]+y^{e}(n-i(\cdot)) \\
\dot{\omega} & =\omega \kappa\left[\left(1-\kappa_{p}\right) \beta_{w}\left(\frac{y}{x l}-\bar{e}\right)+\left(\kappa_{w}-1\right) \beta_{p}\left(\frac{y}{y^{p}}-\bar{u}\right)\right] \\
\dot{l} & =l\left[-i_{q}(q-1)-i_{u}\left(\frac{y}{y^{p}}-\bar{u}\right)\right]
\end{aligned}
$$

possesses an asymptotically stable steady state if $\beta_{w}$ is sufficiently small.

Proof: See the appendix.

Theorem 4 We assume that the economy is a consensus based one, so that labor and capital reach agreement with respect to the scala mobile principle in the $d y$ namics of money wages. Assume also that they agree that additional money wage increases should be small in the boom (when $u-\bar{u}>0$ ) and vice versa in the recession. This makes the steady state of the 5D subdynamics asymptotically stable.

Thus we see that the consensus between capital and labour described in Theorem 4, which is essentially a corporatist income policy, can bring advantages to both parties. It has the additional benefit of simplifying the process about the general level of money wages.

Now we let the parameter $\beta_{n}$ become positive and we can assert:

Proposition 5 Assume that the assumptions of the Proposition 4 hold, but let the parameter $\beta_{n}$ take positive values. Then, the dynamical system becomes

$$
\begin{aligned}
\dot{m} & =m \mu-m\left(\kappa\left[\beta_{p}\left(\frac{y}{y^{p}}-u\right)+\kappa_{p} \beta_{w}\left(\frac{y}{x l}-\bar{e}\right)\right]+\pi_{o}^{c}+i(\cdot)\right) \\
\dot{b}^{l}= & \frac{1}{p_{b}}\left(g-t_{c}-\tau_{w} \omega \frac{y}{x}-\mu m\right)-b^{l}\left(\kappa\left[\beta_{p}\left(\frac{y}{y^{p}}-u\right)+\kappa_{p} \beta_{w}\left(\frac{y}{x l}-\bar{e}\right)\right]+\pi_{o}^{c}+i(\cdot)\right), \\
\dot{y}^{e}= & \beta_{y^{e}}\left[c+i(\cdot)+\operatorname{det} J+g-y^{e}\right]+y^{e}(n-i(\cdot)), \\
\dot{\omega}= & \omega \kappa\left[\left(1-\kappa_{p}\right) \beta_{w}\left(\frac{y}{x l}-\bar{e}\right)+\left(\kappa_{w}-1\right) \beta_{p}\left(\frac{y}{y^{p}}-\bar{u}\right)\right] \\
\dot{l}= & l\left[-i_{q}(q-1)-i_{u}\left(\frac{y}{y^{p}}-\bar{u}\right)\right], \\
\dot{v}= & y-y^{d}-i(\cdot) v, \\
& \quad \text { and possesses an asymptotically stable steady state if } \beta_{n} \text { is sufficiently small. }
\end{aligned}
$$


Proof: The Jacobian of the system (56) is given by

$$
J^{(6)}=\left(\begin{array}{cc}
J_{i, j}^{(5)} & \operatorname{col} 5 \\
\text { row } 5 & -\beta_{n}-n+\left(s_{c}-\tau_{w}\right) \omega \frac{\beta_{n}}{x}-(v+1) \frac{\partial i(\cdot)}{\partial v}
\end{array}\right)
$$

when

$$
\begin{aligned}
& \text { col } 5=
\end{aligned}
$$

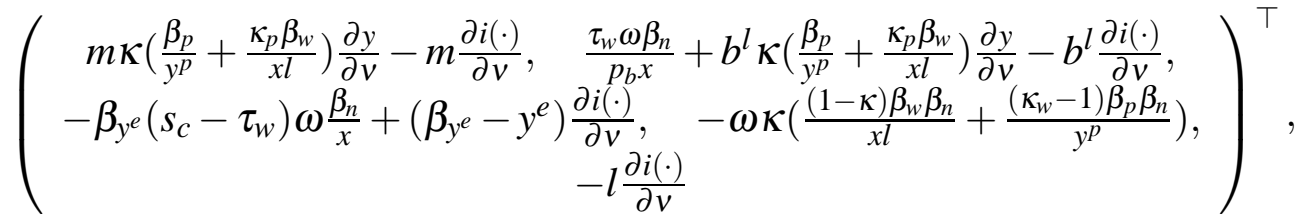

$$
\begin{aligned}
& \text { row } 5= \\
& \left(-(v+1) \frac{\partial i(\cdot)}{\partial m}, \quad-(v+1) \frac{\partial i(\cdot)}{\partial b^{l}}, \quad \frac{\partial y}{\partial y^{e}}-\frac{\partial c}{\partial y^{e}}-(v+1) \frac{\partial i(\cdot)}{\partial y^{e}},\right. \\
& \left.\frac{\left(\tau_{w}-s_{c}\right) y}{x}-(v+1) \frac{\partial i(\cdot)}{\partial \omega}, \quad 0\right) \text {. }
\end{aligned}
$$

With $\beta_{n}=0$ the last column consists of zeros except for $J_{6,6}^{(6)}$ which is $-n$. Thus the eigenvalues of $J^{(6)}$ are $-n$ and the five eigenvalues of $J^{(5)}$ which we have shown to have negative real part. A sufficiently small increase in $\beta_{n}$ will not alter the sign of the eigenvalues.

Theorem 5 For the $6 D$ dynamical system (56) the Metzlerian feedback between expected sales and output is given by

$$
y=\left(1+\alpha_{n^{d}}\left(n+\beta_{n}\right)\right) y^{e}-\beta_{n} v .
$$

This static relationship implies that lowering production via $\alpha_{n^{d}}$ or by cautious inventory adjustment through $\beta_{n}$ (or both) can tame the Metzlerian output accelerator.

We do not introduce any regulation of this Metzlerian sales-inventory adjustment process, but simply assume that this inventory accelerator process is of a secondary nature in the business fluctuations generated by the dynamics, in particular if the control of the Harrodian goods market accelerator of Theorem 1 is working properly.

Next we allow the parameter $\beta_{\pi^{c}}$ to become positive hence obtaining a 7D system about which we have 
Proposition 6 Assume that all assumptions of Proposition 5 are fulfilled, but in addition allow $\beta_{\pi^{c}}$ take positive values. Then the dynamical system

$$
\begin{aligned}
\dot{m} & =m \mu-m\left(\kappa\left[\beta_{p}\left(\frac{y}{y^{p}}-u\right)+\kappa_{p} \beta_{w}\left(\frac{y}{x l}-\bar{e}\right)\right]+\pi^{c}+i(\cdot)\right) \\
\dot{b}^{l} & =\frac{1}{p_{b}}\left(g-t_{c}-\tau_{w} \omega \frac{y}{x}-\mu m\right)-b^{l}\left(\kappa\left[\beta_{p}\left(\frac{y}{y^{p}}-\bar{u}\right)+\kappa_{p} \beta_{w}\left(\frac{y}{x l}-\bar{e}\right)\right]+\pi^{c}+i(\cdot)\right) \\
\dot{y}^{e} & =\beta_{y^{e}}\left[c+i(\cdot)+\delta+g-y^{e}\right]+y^{e}(n-i(\cdot)) \\
\dot{\omega} & =\omega \kappa\left[\left(1-\kappa_{p}\right) \beta_{w}\left(\frac{y}{x l}-\bar{e}\right)+\left(\kappa_{w}-1\right) \beta_{p}\left(\frac{y}{y^{p}}-\bar{u}\right)\right] \\
\dot{l} & =l\left[-i_{q}(q-1)-i_{u}\left(\frac{y}{y^{p}}-\bar{u}\right)\right] \\
\dot{v} & =y-y^{d}-i(\cdot) v \\
\dot{\pi}^{c} & =\alpha \beta_{\pi^{c}} \kappa\left[\beta_{p}(u-\bar{u})+\kappa_{p} \beta_{w}(e-\bar{e})\right]+(1-\alpha) \beta_{\pi^{c}}\left(\mu-n-\pi^{c}\right),
\end{aligned}
$$

possesses a local asymptotically stable steady state if $\beta_{\pi^{c}}$ is sufficiently small.

Proof: The Jacobian of the 7D dynamical system (6) can be written as

$$
J^{(7)}=\left(\begin{array}{cc}
J_{i, j}^{(6)} & \operatorname{col} 7 \\
\operatorname{row} 7 & -(1-\alpha) \beta_{\pi^{c}}
\end{array}\right)
$$

when

$$
\begin{gathered}
\text { row } 7=\left(0,0, \alpha \beta_{\pi^{c}} \kappa\left(\frac{\beta_{p}}{y^{p}}+\frac{\kappa_{p} \beta_{w}}{x l}\right) \frac{\partial y}{\partial y^{e}}, 0,-\alpha \beta_{\pi^{c}} \kappa \kappa_{p} \beta_{w} \frac{y}{x l^{2}}\right. \\
\left.\alpha \beta_{\pi^{c}} \kappa\left(\frac{\beta_{p}}{y^{p}}+\frac{\kappa_{p} \beta_{w}}{x l}\right) \frac{\partial y}{\partial v},-(1-\alpha) \beta_{\pi^{c}}\right), \\
\operatorname{col} 7=\left(-m,-b^{l}, 0,0,0,0,-(1-\alpha)\right)^{\top} .
\end{gathered}
$$

When $\beta_{\pi^{c}}=0$ row 7 consists of zeros, so that the matrix has six eigenvalues with negative real part which are identical to those of the system in Proposition 5 and one eigenvalue of zero. Again we must show that the determinant is negative if $\beta_{\pi^{c}}$ is positive. Multiplying the first row of $\operatorname{det} J^{(7)}$ by $\alpha \beta_{\pi^{c}} / m$ and the fifth row by $-\alpha \beta_{\pi^{c}} / l$ and adding both new rows to the seventh row we get a new seventh row consisting of zeros and a negative entry of $-\beta_{n}$ at the seventh column. All other entries are unchanged by those row operations. According to the proof of Proposition 5 the upper left $6 \times 6$ matrix displays a positive determinant, hence the determinant of $J^{(7)}$ must be negative. 
Theorem 6 Assume that the business cycle is controlled in the way we have described it so far in Theorems 2, 3, 4 and 5. Suppose moreover that the aggregate expectations of price inflation are of the form

$$
\dot{\pi}^{c}=\beta_{\pi^{c}}\left(\alpha \hat{p}+(1-\alpha)(\mu-n)-\pi^{c}\right) ;
$$

then choosing $\alpha$ sufficiently small guarantees the applicability of the preceding proposition.

The economy will thus exhibit damped fluctuations if the parameter $\alpha$ is chosen sufficiently small, which is possible if the business cycle is damped and actual inflation (here only generated by the market for goods) ${ }^{15} \hat{p} \sim \beta_{p}(u-\bar{u}) /(1-$ $\left.\kappa_{p}\right)+\pi^{c}$ is moderate, so that firms are putting sufficient weight on the inflationary climate and not on current wage inflation. A stronger orientation of the change in the inflationary climate on a return to the steady state rate of inflation thus helps to stabilize the economy. Alternatively, the adjustment speed of the inflationary climate may be assumed to be sufficiently small, corresponding to what we have already assumed on the wage price spiral in the preceding theorems.

Note that the consideration of expectations formation on financial markets is still ignored (in fact it is assumed as static). It is however obvious that an enlargement of the dynamics by these expectations does not destroy the stability properties if only fundamentalists are active, since this enlarges the Jacobian solely by a negative entry in its diagonal. Continuity then implies that a portion of chartists that is relatively small as compared to fundamentalists will also preserve the damped fluctuations that we have shown to exist in the above sequence of propositions.

Note that we have excluded the dynamics of the expectations of financial asset prices (as have Asada et al. (2011)). With regard to the dynamic laws for the expectations of equity and bond price inflation we can state.

Proposition 7 Allow the adjustment speeds of the fundamentalist expectations towards the actual growth rate of bond prices $\left(\beta_{\pi_{b f}}\right)$ and equity prices $\left(\beta_{\pi_{e f}}\right)$ to be positive (but keep the corresponding parameters of chartists at zero), then the dynamical system still displays an asymptotically stable steady state.

Proof: The law of motion of the expectations of the fundamentalists with respect to the growth rate in equity prices given by equation (45) shows that there is always a stable motion of expectations towards the steady state, since no other variable affects this motion. The same is true for the law of motion of the fundamentalists

\footnotetext{
${ }^{15}$ Here the symbol $\sim$ means "is dominated by'.
} 
expectation about the inflation rate of long term bonds in equation (43). Adding these two laws of motion to the dynamical system means that the Jacobian will expand by two columns and two rows to become a 9D system. The rows will contain only zeros with the exception of the main diagonal, which will be negative. These are the negative values of the adjustment speeds. The two new eigenvalues are thus the negative adjustment speeds while the other eigenvalues are the same as those of $J^{(7)}$. The latter have negative real values as we have seen in Proposition 6. Hence the asymptotically stable steady state is preserved.

In order to have the result of Proposition 7 enforced by policy action, independently of the size of the chartist population, we introduce Tobin type taxes on the capitals gains of equities and long-term bonds in the form

$$
\begin{aligned}
& \dot{\pi}_{e c}=\beta_{\pi_{e c}}\left(\tau_{e} \hat{p}_{e}-\pi_{e c}\right), \\
& \dot{\pi}_{b c}=\beta_{\pi_{b c}}\left(\tau_{b} \hat{p}_{b}-\pi_{b c}\right) .
\end{aligned}
$$

Such taxes could be monitored through a corresponding tax declaration scheme which not only taxes capital gains, but also subsidizes capital losses (and thus does not entirely disadvantage the asset holders of the model).

Theorem 7 For Tobin tax parameters $\tau_{e}, \tau_{b}$ chosen sufficiently large (but below $100 \%)$ the damped business fluctuations remain damped.

The financial market accelerator can therefore be tamed through the introduction of appropriate levels of Tobinian capital gains taxation rules. Note however that this rule introduces a new sector into the economy, one which accumulates or decumulates reserve funds $(R)$ according to the rule

$$
\dot{R}=\tau_{e} \dot{p}_{e} E+\tau_{b} \dot{p}_{b} B^{l} .
$$

In order to keep again the laws of motion of the economy unchanged (and so allow the application of the above stability propositions) we thus assume that this new sector is independent of the other public institutions. The steady state value $\rho^{o}$ of the funds of the new sector expressed per value unit of capital $p K$ (to be extended to long-term bonds) is

$$
\rho^{o}=(R / p K)^{o}=\tau_{e}(\mu-n) / \mu<1,
$$

which follows from the law of motion

$$
\hat{\rho}=\hat{R}-\hat{p}-\hat{K}=\frac{\dot{R}}{R} \frac{R}{p K}-\hat{p}-\hat{K}
$$

since there holds $\hat{p}-\hat{K}=\mu$ and $\hat{E}=n, q=1, \hat{p}_{e}=\hat{p}$ in the steady state. It is assumed that the reserves of this institution are sufficiently large so that they will 
not become exhausted during the phases of damped business fluctuations generated by the model.

The stability results of the propositions are intuitively very appealing since they basically state that the wage-spiral must be fairly damped, the Keynesian dynamic multiplier be stable and not too much distorted by the emergence of Metzlerian inventory cycles, that the Harrodian knife-edge growth accelerator is weak, and that inflationary and capital gains expectations are fundamentalist in orientation and money demand is subject to small transaction costs and fairly unresponsive to rate of return changes on financial assets (that is money demand is not too close to a liquidity trap).

We have shown in the above theorems the result that independently conducted countercyclical fiscal policy can limit the fluctuations on the goods market, that an appropriate consensus between capital and labor can tame the wage-price spiral and that a Tobin tax can tame the financial market accelerator. Metzlerian inventory dynamics and fluctuations in the inflationary climate that is surrounding the economy may then also be weak and thus not endanger asymptotic stability.

For the chartists we merely conjecture (but do not prove), that positive but small adjustment speeds of their expectations on asset prices growth rates will preserve asymptotic stability. It is difficult to prove such a conjecture since the dynamics is much more complex than that for the fundamentalists, since there are complicated feedback channels involved.

Asada et al. (2010) have considered a basic version of a KMG-Tobin model with a portfolio structure consisting of the assets money, fix-price bonds (with a variable rate of interest) and equities. Equities were therefore the only risk-bearing asset. The asset markets determined through their equilibrium conditions the shortterm rate of interest and Tobin's $q$, the latter in turn providing the only link to the real part of the economy by entering the investment decisions of firms. Monetary policy must therefore have an impact of Tobin's $q$ in order to influence economic activity and hence the rate of inflation. In the model of Asada et al. (2010) it is also very natural to assume as asset demand structure (around the steady state) of the form

$$
\begin{aligned}
M & =M^{d}=f_{m}(i) W_{c}^{n}, \\
B & =B^{d}=f_{b}\left(i, r_{e}^{e}\right) W_{c}^{n}, \\
p_{e} E & =p_{e} E^{d}=f_{e}\left(r_{e}^{e}\right) W_{c}^{n} .
\end{aligned}
$$

We here have two assets that are perfectly liquid, $M$ and $B$, where $M$ is only used for transactions purposes and thus subject to a simple Baumol type of inventory decision rule; see Baumol (1952). However there is the possibility for the central bank to influence the economy via an interest rate policy. In Asada 
et al. (2010) such a policy did not affect the real part of the economy, since it only influenced the composition of $M+B$, but not its size. A direct influence on Tobin's $q$ was therefore excluded in that analysis, since such a policy was assumed to not change the expectations of the agents that act on the financial market. Only a policy function that reacts countercyclically to the deviations of Tobin's $q$ from its steady state value can thus contribute to the stabilization of the business cycle. But central banks may be reluctant to adopt such a policy, a rule of quantitative easing type in fact, which is debated in the literature, see Palley (2011). As suggested by Keynes, central banks must at least be prepared to act on the long end of the bond market in order to effectively control economic activity.

In this paper we have replaced short- term bonds by long-term bonds and considered the asset demands

$$
\begin{aligned}
M_{2} & =M_{2}^{d}=f_{m}\left(r_{b}^{e}, r_{e}^{e}\right) W_{c}^{n} \\
p_{b} B^{l} & =p_{b} B^{l d}=f_{b^{l}}\left(r_{b}^{e}, r_{e}^{e}\right) W_{c}^{n} \\
p_{e} E & =p_{e} E^{d}=f_{e}\left(r_{b}^{e}, r_{e}^{e}\right) W_{c}^{n} .
\end{aligned}
$$

We have two risk-bearing assets, only one of which (in aggregate form) is perfectly liquid and thus can be the object of hoarding (liquidity preference). This is definitely an improvement in comparison to Asada et al. (2010). Central banks are assumed now to trade at least in long-term bonds (if not even equities), since trade in short-term bonds may not be effective. It may therefore be worthwhile to consider short-term bonds explicitly in order to see what an interest rate policy on the short end of the market can achieve.

A central conclusion of the present section is that the stability properties of the model may be too weak to give rise to damped oscillations around the balanced growth path by themselves, and thus the policy actions discussed may be necessary in order to stabilize such an unstable economy.

\section{Monetary Policy}

We have so far only considered the portfolio choice of asset holders between $M_{2}, B^{l}, E$, but not their demand for money $M$ and short-term bonds $B$ separately. This second step in the portfolio management process of asset holders is however a very simple one, since it only concerns their cash management, their intentions to hold central bank money $M$ versus their holding of saving deposits $B_{c}$. We thus simply add the relationships (the reader should keep in mind that the quantity $B_{b}$ is not available to the public, but held by the central bank)

$$
M_{2}=M+B_{c} \equiv M^{d}(i)+B_{c}^{d}(i), \quad M_{i}^{d}=-B_{c i}^{d}<0 .
$$


Equation (60) states that asset holders intend to reduce their cash holdings in the case of an increasing rate of interest on short-term bonds. Assuming an interest rate policy rule as characterizing the monetary policy of the central bank then simply implies that the central bank has to adjust to the cash and short-term bond composition that is desired by the asset holders at this rate of interest. But this adjustment process does not change anything in the rest of the financial markets and also nothing in the real sector or the economy.

Monetary policy is therefore completely ineffective in our model if it acts solely on the short term end of the asset markets, unless one assumes that agents mistakenly consider monetary policy as being effective and interpret a changing rate of interest as a change of the assumed state of confidence of both themselves and the central bank. But this may be a very unreliable channel for such a monetary policy, since it is based on interpretations of the prevailing situation that may be subject to sudden and uncontrollable regime switches.

We shall thus assume for simplicity an interest rate peg of the central bank and a given composition of the money holdings $M_{2}=M+B_{c}$ of asset holders. Concerning the supply of new money we moreover assume a constant growth rate $\mu$, an assumption which we also apply to the growth rate of short-term government bonds $B$. This gives rise to the flow conditions

$$
\dot{M}=\mu M=\dot{B}_{b}, \quad p_{b} \dot{B}^{l}=p G+B^{l}+i B_{c}-p T-\dot{B}, \quad \dot{B}=\mu B,
$$

with $B_{b}, B_{c}$ being the holdings of short-term government debt of the central bank and the asset holders respectively. Thus

$$
p_{b} \dot{B}^{l}=p\left(G-T_{c}\right)-\dot{M}-\dot{B}_{c}=p\left(G-T_{c}\right)-\mu\left(M+B_{c}\right), \quad T_{c}=T-\left(B^{l}+i B_{c}\right) / p
$$

since the growth rate of $B_{c}$ must be equal to that of $B$ in this situation. Note that in deriving the first equation on the last line we have used the result that $\dot{B}_{c}+\dot{B}_{b}=$ $\dot{B}$. This implies the law of motion for the long-term debt of the government (see Proposition 2) now becomes

$$
\dot{b}^{l}=\frac{1}{p_{b}(\cdot)}\left(g-t_{c}-\tau_{w} \omega l^{d}-\mu\left(m+b_{c}\right)\right)-b^{l}\left(\pi_{o}^{c}+i(\cdot)\right) .
$$

Here, the discussion of the expression $m+b$ is simply replaced by $m+$ $b_{c}$, since part of the short-term bonds is now explicitly held by the central bank (which transfers the resulting interest income back into the government sector at each moment in time). This implies that also the asset markets and private wealth are now based on $B_{c}$ in place of $B$, with $\dot{B}=\mu B$ as the new inflow into these asset markets. 
In view of the given structure of the private sector of the economy the following alternative to an interest rate policy rule may now be the best choice as monetary policy for the central bank. Concerning the long-run control of inflation it keeps pursuing the Friedman type of open market policy rule, namely

$$
\dot{M}_{l}=\mu M=\dot{B}_{b}, \quad \mu=\text { constant } .
$$

For the total change in money supply we now however assume on this basis that it obeys the rule $\left(M=M_{l}+M_{s}\right)$

$$
\dot{M}_{s}=\dot{M}-\dot{M}_{l}=p_{e} \dot{E}_{b}, \quad \dot{M}_{s}=-\beta_{m q}\left(q_{o}-q\right)\left(M+B_{c}\right),
$$

where $E_{b}$ denotes the amount of equities held by the central bank. As short-run monetary policy the central bank now trades in equities instead of short-term government debt, in view of the role Tobin's $q$ plays in the investment function of the present model. The assumed rule states that the central bank supplies extra equities on the stock market in a boom and buys equities in a downturn. With this policy it thus aims to make the stock market less volatile.

We therefore now have also to distinguish central bank and private holdings of equities $E=E_{b}+E_{c}$. For total private wealth this gives the defining expression $W_{c}^{n}=M+B_{c}+p_{b} B^{l}+p_{e} E_{c}$ in place of the sum of items we have used so far, so that we get as representation of the equity market in intensive form

$$
q=f_{e}\left(r_{e}^{e}, r_{b}^{e}\right)\left(m+b_{c}+p_{b} b^{l}+q\right) .
$$

The stock of equities held by the asset holders therefore does not enter the dynamics explicitly, but only in the form of Tobin's $q$ as determined by the private part of the asset markets (to be used in the investment function as before).

The above short-term policy of the central bank implies for the law of motion of the money supply $m_{2}=\left(M+B_{c}\right) /(p K)$ the expression ${ }^{16}$

$$
\dot{m_{2}}=\mu m_{2}-\left(\pi^{c}+i(\cdot)\right) m_{2}-\beta_{m q}\left(q_{o}-q\right) m_{2} .
$$

This is the only change in the laws of motion considered, which therefore can again be analyzed as in the preceding section.

\footnotetext{
${ }^{16}$ Note that this was formerly denoted by $m$.
} 
Theorem 8 The now augmented 3D subdynamics of the full 9D dynamics:

$$
\begin{aligned}
\dot{m_{2}} & =\mu m_{2}-\left(\pi_{o}^{c}+i(\cdot)\right) m_{2}-\beta_{m q}\left(q_{o}-q\right) m_{2}, \\
\dot{b}^{l} & =\frac{1}{p_{b}(\cdot)}\left(g-t_{c}-\tau_{w} \omega l^{d}-\mu m_{2}\right)-b^{l}\left(\pi_{o}^{c}+i(\cdot)\right), \\
\dot{y}^{e} & =\beta_{y^{e}}\left(y^{d}(\cdot)-y^{e}\right)+(n-i(\cdot)) y^{e},
\end{aligned}
$$

can be additionally stabilized (by increasing the parameter range where damped oscillations are established and by making the originally given damped oscillations even less volatile) by increasing the parameter value $\beta_{m q}$ of the new term $-\beta_{m q}\left(q-q^{o}\right) m_{2}$ in the law of motion for real balances, if anticyclical fiscal policy is sufficiently active to make the dynamic multiplier process a stable one (by neutralizing the Harrodian investment accelerator) and if the savings rate $s_{c}$ of asset holders is sufficiently close to one (which allows one to ignore effects from taxation on the consumption of asset holders).

Proof: ${ }^{17}$ From the comparative static exercises we know that Tobin's $q$ depends positively on $m_{2}$ and also positively on $b^{l}$ if it is assumed in the latter case that the equity market is sufficiently tranquil (if the parameter $\alpha_{e 1}$ is sufficiently small). Moreover, the price of perpetuities $p_{b}$ always depends positively on $m_{2}$ and negatively on $b^{l}$.

The Routh-Hurwitz polynomial of the Jacobian matrix of the given extension of the original model is now augmented through the assumed stock market policy by the principal minors that are obtained from the additional matrix of the dynamical equations

$$
\begin{aligned}
\dot{m}_{2} & =-\beta_{m q}\left(q\left(m_{2}, b^{l}, r_{k}^{e}\right)-q^{o}\right) m_{2}, \\
\dot{b}^{l} & =\frac{1}{p_{b o}(\cdot)}\left(g-t_{c}-\tau_{w} \omega l^{d}-\mu m_{2}\right)-b^{l}\left(\pi_{o}^{c}+i(\cdot)\right), \\
\dot{y}^{e} & =\beta_{y^{e}}\left(y^{d}(\cdot)-y^{e}\right)+(n-i(\cdot)) y^{e},
\end{aligned}
$$

which only differs from the original one in its first row (as far as the formation of the Jacobian matrix is concerned). This row can be used to eliminate the $i_{q}(\cdot)$ term in the $i(\cdot)$ function when calculating the principal minors of this additional Jacobian matrix. Thereby the last row in the Jacobian becomes of the form $(0,0,-)$.

\footnotetext{
${ }^{17}$ Here we merely give a sketch of the proof as lack of space prevents us from giving full details, which are similar in spirit to the other proofs that we have already given.
} 
We had already shown that

$$
\frac{\partial q}{\partial m}>\frac{\partial q}{\partial\left(p_{b} b^{l}\right)},
$$

which implies the the upper minor of order $2, J_{3}, J_{3,3}^{(3)}$ of the laws of motion is positive, implying that the 3D determinant is negative. The other (additional) principal minors of order 2 are also positive and the trace is clearly negative. From these results one then easily gets that the Routh-Hurwitz coefficients of the characteristic polynomial of the augmented Jacobian exceed the original ones, while the negative of the determinant of the Jacobian is dominated by the product $\operatorname{tr}\left(J^{(3)}\right)\left(J_{1}^{(3)}+\right.$ $\left.J_{2}^{(3)}+J_{3}^{(3)}\right)$, so that the final Routh-Hurwitz condition $\operatorname{tr}\left(J^{(3)}\right)\left(J_{1}^{(3)}+J_{2}^{(3)}+J_{3}^{(3)}\right)-$ $\operatorname{det} J^{(3)}<0$ is obviously also fulfilled.

This theorem is however a conjecture only at the present state of the investigation of our KMG-Tobin model, since we can only prove it for the moment when we assume that the price of long-term bonds remains at their steady state value. However if one integrates the fluctuations of the price of perpetuities into this stability discussion one obtains an additional term in the upper minor of order 2 of the form:

$$
\left(\begin{array}{ll}
- & - \\
- & +
\end{array}\right)
$$

which clearly adds instability to the dynamics.

Therefore, the consideration of long-term government bonds in the dynamic law of motion for government debt adds centrifugal forces to the considered dynamical system, since in particular there is now a positive feedback between the level of long-term debt and its rate of change (per unit of capital). We stress that this is a price effect and not a quantity effect in the dynamics of the government budget equation, since the accumulation of long-term debt lowers its price and therefore leads to more flow demand of the government for such debt. One may hope that this effect is dominated by the many stabilizing effects we have discussed in the preceding section. If not, the government has to change the financing of its debt into a direction which eliminates such cumulative forces.

We note that the destabilizing minor of order 2 was not present in the model with only fix-price bonds as in paper by Asada et al. (2010). Moreover, stability is less likely in the private sector of the economy now, due to the assumed existence of one risky intermediate asset between fix-price bonds and equities. This remark however only concerns the 3D structure of the model, while the stability proposition built on the initial scenario - on the role of corporate income policies, fiscal policy and the inventory cycle - holds in the model extended by long-term government debt as well. The most crucial feature of the considered model is however the 
interaction between financial markets and the $q$-theory of investment behavior of the model.

\section{Conclusions}

Summing up, it is not the individual behavior of economic agents (firms, households, institutions) but rather the interconnectedness of agents and sectors that produces the stabilizing or destabilizing feedback effects. Left to itself, the macroeconomy has experienced large boom-bust cycles, with extensive externalities when the bubble has burst. In the context of our proposed model we would argue that boombust cycles can be dampened. More specifically, in terms of policy, we have shown that countercyclical labor market and fiscal policies, with a tranquilized wage-price spiral, a Tobin tax on capital gains and the implementation of a Tobin rule in place of a Taylor rule could be - taken together - powerful means to make the business cycle not only less volatile, but damped and maybe also convergent to some balanced growth path of the economy. Besides demand management by a fiscal authority, wage management through cooperation between capital and labor, we must have monetary policies that concentrate on financial markets - here represented by money, long term bonds and equity - in order to dampen business cycles on the macro level by means of policies of buying and selling financial assets.

We should point out that the real wage dynamics are independent of the law of motion of inflationary climate expectations. In fact, the main results precede the inclusion of $\pi^{c}$ into the considered cascade of stable matrices.

Our model has allowed for non-conventioanl monetary policy in the sense of buying assets in the recession and selling them in the boom. The stabilizing effects then come through the equity market and Tobin's $q$. What we have studied here is basically the Bernanke Fed policy of "quantitative easing", where we consider however only the buying and selling of equity assets, not bonds. Though the latter was also part of the quantitative easing policy, we have neglected this in the current paper. This will be subject of future research.

\section{Glossary: Summary of Notation}

Steady state values are indicated by a superscript ' $o$ '. A dot over a variable $x=x(t)$ denotes the time derivative, a caret its growth rate; $\dot{x}=d x / d t, \hat{x}=\dot{x} / x$.

As far as possible, the notation tries to follow the logic of using capital letters for level variables and lower case letters for variables in intensive form, or 
for constant (steady state) ratios. Greek letters are most often constant coefficients in behavioural equations (with, however, the notable exceptions being $\pi, \omega$ ).

A. Statically or dynamically endogenous variables:

$Y$

$Y^{d}$

$Y^{p}$

$Y^{e}$

$u=Y / Y^{p}$

$K$

$L$

$L^{d}$

$e=L^{d} / L$

C

I

$\mathscr{I}$

$N$

$N^{d}$

$i$

$p$

$p_{e}$

$p_{b}$

$q$

$\pi_{b}=\hat{p}_{b}^{e}$

$\pi_{e}=\hat{p}_{e}^{e}$

$T^{n}(T)$

G

$r^{e}$

$r_{b}^{e}$

$r_{e}^{e}$

$r_{k}^{e}$

$w$

$\omega=w / p$

$\pi^{c}$

B

$B^{l}$

E

$W^{n}, W$

$n$

$\hat{z}$

$t^{n}$
Output of the domestic good

Aggregate demand for the domestic good

Potential output of the domestic good

Expected sales for the domestic good

Rate of capacity utilisation of firms

Capital stock

Labor supply

Total employment of the employed

Rate of employment ( $\bar{e}$ the employment-complement of the NAIRU)

Total goods consumption

Gross business fixed investment

Planned inventory investment

Actual inventories

Desired inventories

Nominal short-term rate of interest (price of long-term bonds $p_{b}=1$ )

price level

Price of equities

Price of long-term bonds

Tobin's $q=$ the value of equity over the value of capital $=p_{e} E /(p K)$

expected appreciation in the price of long-term domestic bonds

expected appreciation in the price of equities

Nominal (real) taxes

Real government expenditure

Expected short-run rate of profit of firms

The expected rate of return on long terms bonds

The expected rate of return on equity

The expected rate of return on capital

Nominal wages before taxes

real wages

Expected rate of inflation or inflation climate

Stock of domestic short-term bonds (index d: demand)

Stock of domestic long-term bonds. (index d: demand)

Equities (index d: demand)

Nominal and real domestic wealth

Natural growth rate of the labor force (adjustment towards $\tilde{n}$ )

Rate of Harrod neutral technical change

total taxes per value unit of capital

B. Parameters of the model

$\delta$

$\beta_{x}$

$\bar{e}$
Depreciation rate of the capital stock of firms

All $\beta$-expressions (adjustment speeds)

NAIRU employment rate (NAIRE) 


$\begin{array}{ll}\bar{u} & \text { Normal rate of capacity utilisation of firms } \\ \kappa_{w}, \kappa_{p} & \text { Weights of short- and long-run inflation }\left(\kappa_{w} \kappa_{p} \neq 1\right) \\ \kappa & =\left(1-\kappa_{w} \kappa_{p}\right)^{-1} \\ y^{p} & \text { Output-capital ratio }\end{array}$

\section{APPENDIX}

\section{Proof of Proposition 2}

The Jacobian of the 3D system (53) is

$$
J^{(3)}=\left(\begin{array}{ccc}
-m \frac{\partial i(\cdot)}{\partial m} & -m \frac{\partial i(\cdot)}{\partial b^{l}} & -m \frac{\partial i(\cdot)}{\partial y^{e}} \\
\frac{\partial p_{b}}{\partial m}(\cdot)-\frac{\mu}{p_{b}}-b^{l} \frac{\partial i(\cdot)}{\partial m} & \frac{\partial p_{b}}{\partial b^{l}}(\cdot)-\mu-b^{l} \frac{\partial i(\cdot)}{\partial b^{l}} & -\frac{1}{p_{b}} \tau_{w} \omega \frac{1}{x} \frac{\partial y}{\partial y^{e}}-b^{l} \frac{\partial i(\cdot)}{\partial y^{e}} \\
\left(\beta_{y^{e}}-y^{e}\right) \frac{\partial i(\cdot)}{\partial m} & \left(\beta_{y^{e}}-y^{e}\right) \frac{\partial i(\cdot)}{\partial b^{l}} & \beta_{y^{e}}\left(\frac{\partial c}{\partial y^{e}}-1\right)+\left(\beta_{y^{e}}-y^{e}\right) \frac{\partial i(\cdot)}{d y^{e}}
\end{array}\right),
$$

with $i(\cdot)=-\frac{1}{p_{b}^{2}}\left(g-t_{c}-\tau_{w} \omega l^{d}-\mu m\right)$. In steady state we can conclude from the law of motion of $b^{l}$ that $i(\cdot)=-\frac{1}{p_{b}} b^{l} \mu$. We shall prove local asymptotic stability by means of the necessary and sufficient Routh-Hurwitz conditions for a dynamical system of three differential equations, namely

$$
\begin{aligned}
\operatorname{tr} J^{(3)} & <0, \\
\operatorname{det} J^{(3)} & <0, \\
\left|J_{M, 1}^{(3)}\right|+\left|J_{M, 2}^{(3)}\right|+\left|J_{M, 3}^{(3)}\right| & >0, \\
\operatorname{tr}\left(J^{(3)}\right)\left(J_{M, 1}^{(3)}+J_{M, 2}^{(3)}+J_{M, 3}^{(3)}\right)-\operatorname{det} J^{(3)} & <0 .
\end{aligned}
$$

with $J_{M, i}^{(3)}$ being the principal minor of the matrix entry $J_{i i}^{(3)}$. To prove (62) we calculate:

$$
\begin{aligned}
\operatorname{tr} J^{(3)} & =-m \frac{\partial i(\cdot)}{\partial m}+\frac{\partial p_{b}}{\partial b^{l}}(\cdot)-\mu-b^{l} \frac{\partial i(\cdot)}{\partial b^{l}}+\beta_{y^{e}}\left(\frac{\partial c}{\partial y^{e}}-1\right)+\left(\beta_{y^{e}}-y^{e}\right) \frac{\partial i(\cdot)}{\partial y^{e}} \\
& =-m i_{q} \frac{\partial q}{\partial m}-b^{l} i_{q} \frac{\partial q}{\partial b^{l}}-\frac{\partial p_{b}}{\partial b^{l}} \frac{1}{p_{b}} b^{l} \mu-\mu+\beta_{y^{e}}\left(\frac{\partial c}{\partial y^{e}}+\frac{\partial i(\cdot)}{\partial y^{e}}-1\right)-y^{e} \frac{\partial i(\cdot)}{\partial y^{e}} .
\end{aligned}
$$

The first two terms of the sum can be recalculated as

$$
-m i_{q} \frac{\partial q}{\partial m}-b^{l} i_{q} \frac{\partial q}{\partial b^{l}}=-\frac{1}{\operatorname{det} J^{(2)}} i_{q}\left(\alpha_{b^{l} 1} \frac{1}{p_{b}^{2}} m q+\alpha_{m 1} \frac{1}{p_{b}^{2}} p_{b} b^{l} q+\frac{m b^{l} q}{m+p_{b} b^{l}+q}\right) .
$$

We have seen in Section 5 that $\operatorname{det} J^{(2)}$ is positive, ${ }^{18}$ hence $-m i_{q} \frac{\partial q}{\partial m}-b^{l} i_{q} \frac{\partial q}{\partial b^{l}}<0$.

\footnotetext{
${ }^{18}$ See the sentence below equation (52).
} 
The next two parts of the sum in the trace are $-\frac{\partial p_{b}}{\partial b^{l}} \frac{1}{p_{b}} b^{l} \mu-\mu$ which has a negative sign since $-\frac{\partial p_{b}}{\partial b^{l}}\left(1 / p_{b} \mu b^{l}\right)-\mu<0,-\frac{\partial p_{b}}{\partial b^{l}}\left(1 / p_{b} b^{l}\right)-1<0$, and $\frac{1}{\operatorname{det} J^{(2)}}\left(\alpha_{m 2} \frac{r_{k}^{e}}{q^{2}} b^{l}(m+\right.$ $\left.q)+\alpha_{b^{l} 2} \frac{r_{k}^{e}}{q^{2}} b^{l} m+\frac{m b^{l}}{m+p_{b} b^{l}+q}\right)<1$. The positive denominator exceeds the positive numerator as one can see by means of equation (52), and hence the inequality holds.

Analogously to the model with short term bonds one can show that the term $\beta_{y^{e}}\left(\frac{\partial c}{\partial y^{e}}-1\right)$ is negative. Adding $\beta_{y^{e}} \frac{\partial i(\cdot)}{\partial y^{e}}$ we get $\beta_{y^{e}}\left(\frac{\partial c}{\partial y^{e}}+\frac{\partial i(\cdot)}{\partial y^{e}}-1\right)$. To determine sign of $\frac{\partial i(\cdot)}{\partial y^{e}}$, we calculate

$$
\frac{\partial i(\cdot)}{\partial y^{e}}=i_{q} \frac{\partial q}{\partial y^{e}}+i_{u} \frac{1}{x} \frac{\partial y}{\partial y^{e}}=i_{q} \frac{\partial q}{\partial r_{k}^{e}} \frac{\partial r_{k}^{e}}{\partial y^{e}}+i_{u} \frac{1}{x} \frac{\partial y}{\partial y^{e}}
$$

We know already from Section 5 that $\frac{\partial q}{\partial r_{k}^{e}}>0$. The other partial derivatives are also positive, which is easy to verify by using the definitions of $y$ and $r_{k}^{e}$. To guarantee a negative trace, although the adjustment speed $\beta_{y^{e}}$ may be very large, the term $\frac{\partial c}{\partial y^{e}}-\frac{\partial i(\cdot)}{\partial y^{e}}-1$ must stay negative. The condition that $i_{u}$ is sufficiently small limits the influence of the second part of the sum in (66) and choosing $\alpha_{b^{l} 2}$ and $\alpha_{m 2}$ sufficiently small makes the first part of the sum after the second equality sign in (66) sufficiently small. ${ }^{19}$ The only positive entry then is $y^{e} \frac{\partial i(\cdot)}{\partial y^{e}}$. For every value of this last element of the sum we know that there are sufficiently large values for $\beta_{y^{e}}$ that preserve the negative sign of the trace. Hence a sufficiently large adjustment speed of the actual production to expected demand assures that the first RouthHurwitz condition (62) is met.

The third Routh-Hurwitz condition (64) concerns the sum of the secondary principal minors denoted by $\left|J_{M, 1}^{(3)}\right|,\left|J_{M, 2}^{(3)}\right|,\left|J_{M, 3}^{(3)}\right|$, of the Jacobian $J^{(3)}$. We first calculate

$$
\left|J_{M, 1}^{(3)}\right|=\left|\begin{array}{cc}
-\frac{\partial p_{b}}{\partial b^{l}} \frac{1}{p_{b}} b^{l} \mu-\mu-b^{l} i_{q} \frac{\partial q}{\partial b^{l}} & -\frac{1}{p_{b}} \tau_{w} \omega \frac{1}{x} \frac{\partial y}{\partial y^{e}}-b^{l} \frac{\partial i(\cdot)}{\partial y^{e}} \\
\left(\beta_{y^{e}}-y^{e}\right) i_{q} \frac{\partial q}{\partial b^{l}} & \beta_{y^{e}}\left(\frac{\partial c}{\partial y^{e}}-1\right)+\left(\beta_{y^{e}}-y^{e}\right) \frac{d i(\cdot)}{\partial y^{e}}
\end{array}\right| .
$$

In order to guarantee that this determinant is positive we require that $-\frac{\partial p_{b}}{\partial b^{l}} \frac{1}{p_{b}} b^{l} \mu-$ $\mu$ be negative, but this result has been shown in the proof that trace $J^{(3)}<0$.

We know that $0<\frac{\partial p_{b}}{\partial b^{l}} \frac{1}{p_{b}} b^{l}<1$. Hence a sufficient condition to have $\left|J_{M, 1}^{(3)}\right|>$ 0 is $\frac{\partial q}{\partial b^{l}}>0$ which is the case if $\alpha_{m 1}$ is sufficiently large as was shown in Section 4.

\footnotetext{
${ }^{19}$ Alternatively to the condition of a small $\alpha_{b^{l 2}}$ and $\alpha_{m 2}$, one may also consider sufficiently small $i_{q}$.
} 
Next we calculate

$$
\begin{aligned}
\left|J_{M, 2}^{(3)}\right| & =\left|\begin{array}{cc}
-m i_{q} \frac{\partial q}{\partial m} & -m \frac{\partial i(\cdot)}{\partial y^{e}} \\
\left(\beta_{y^{e}}-y^{e}\right) i_{q} \frac{\partial q}{\partial m} & \beta_{y^{e}}\left(\frac{\partial c}{\partial y^{e}}-1\right)+\left(\beta_{y^{e}}-y^{e}\right) \frac{\partial i(\cdot)}{d y^{e}}
\end{array}\right| \\
= & \left|\begin{array}{cc}
-m i_{q} \frac{\partial q}{\partial m} & -m \frac{\partial i(\cdot)}{\partial y^{e}} \\
0 & \beta_{y^{e}}\left(\frac{\partial c}{\partial y^{e}}-1\right)
\end{array}\right|,
\end{aligned}
$$

which is positive because $\frac{\partial q}{\partial m}>0$ as shown Section 5. Finally we calculate

$$
\begin{aligned}
\left|J_{M, 3}^{(3)}\right| & =\left|\begin{array}{cc}
-m i_{q} \frac{\partial q}{\partial m} & -m i_{q} \frac{\partial q}{\partial b^{l}} \\
\frac{\partial p_{b}}{\partial m}(\cdot)-\frac{1}{p_{b}} \mu-b^{l} i_{q} \frac{\partial q}{\partial m} & \frac{\partial p_{b}}{\partial b^{l}}(\cdot)-\mu-b^{l} i_{q} \frac{\partial q}{\partial b^{l}}
\end{array}\right| \\
& =\left|\begin{array}{cc}
-m i_{q} \frac{\partial q}{\partial m} & -m i_{q} \frac{\partial q}{\partial b^{l}} \\
\frac{\partial p_{b}}{\partial m}(\cdot)-\frac{1}{p_{b}} \mu & \frac{\partial p_{b}}{\partial b^{l}}(\cdot)-\mu
\end{array}\right|>0 .
\end{aligned}
$$

With all three principal minors positive, we can assert that

$$
\left|J_{M, 1}^{(3)}\right|+\left|J_{M, 2}^{(3)}\right|+\left|J_{M, 3}^{(3)}\right|>0 .
$$

The second Routh-Hurwitz condition (63) reads

$$
\begin{aligned}
& -m i_{q} \frac{\partial q}{\partial m} \quad-m i_{q} \frac{\partial q}{\partial b^{l}} \quad-m \frac{\partial i(\cdot)}{\partial y^{e}} \\
& \frac{\partial p_{b}}{\partial m}(\cdot)-\frac{\mu}{p_{b}}-b^{l} i_{q} \frac{\partial q}{\partial m} \quad \frac{\partial p_{b}}{\partial b^{l}}(\cdot)-\mu-b^{l} i_{q} \frac{\partial q}{\partial b^{l}} \quad-\frac{1}{p_{b}} \tau_{w} \omega \frac{1}{x} \frac{\partial y}{\partial y^{e}}-b^{l} \frac{\partial i(\cdot)}{\partial y^{e}} \\
& \left(\beta_{y^{e}}-y^{e}\right) i_{q} \frac{\partial q}{\partial m} \quad\left(\beta_{y^{e}}-y^{e}\right) i_{q} \frac{\partial q}{\partial b^{l}} \quad \beta_{y^{e}}\left(\frac{\partial c}{\partial y^{e}}-1\right)+\left(\beta_{y^{e}}-y^{e}\right) \frac{\partial i(\cdot)}{d y^{e}} \\
& =\left|\begin{array}{ccc}
-m i_{q} \frac{\partial q}{\partial m} & -m i_{q} \frac{\partial q}{\partial b^{l}} & -m \frac{\partial i(\cdot)}{\partial y^{e}} \\
\frac{\partial p_{b}}{\partial m}(\cdot)-\frac{1}{p_{b}} \mu-b^{l} i_{q} \frac{\partial q}{\partial m} & \frac{\partial p_{b}}{\partial b^{l}}(\cdot)-\mu-b^{l} i_{q} \frac{\partial q}{\partial b^{l}} & -\frac{1}{p_{b}} \tau_{w} \omega \frac{1}{x} \frac{\partial y}{\partial y^{e}}-b^{l} \frac{\partial i(\cdot)}{\partial y^{e}} \\
0 & 0 & \beta_{y^{e}}\left(\frac{\partial c}{\partial y^{e}}-1\right)
\end{array}\right| \\
& =\beta_{y^{e}}\left(\frac{\partial c}{\partial y^{e}}-1\right)\left|J_{M, 3}^{(3)}\right|
\end{aligned}
$$

where $\left|J_{M, 3}^{(3)}\right|$ has been shown above to be positive. We know that $\beta_{y^{e}}\left(\frac{\partial c}{\partial y^{e}}-1\right)$ is negative, so (63) is satisfied.

The fourth Routh-Hurwitz condition in equation (65) is fulfilled for sufficiently large values of $\beta_{y e}$, because $\beta_{y^{e}}$ is quadratic with a positive sign in the first term of the sum, and is only linear in the second term. 


\section{Proof of Proposition 3}

The proof makes use of the continuity of the eigenvalues of a matrix with respect to changes in the entries. The Jacobian of the system (54) can be written as

$$
J^{(4)}\left(\beta_{p}\right)=\left(\begin{array}{cclc}
J_{1,1}^{(3)} & J_{1,2}^{(3)} & J_{1,3}^{(3)}-m \frac{\kappa \beta_{p}}{y^{p}} \frac{\partial y}{\partial y^{e}} & -m \frac{\partial i(\cdot)}{\partial \omega} \\
J_{2,1}^{(3)} & J_{2,2}^{(3)} & J_{2,3}^{(3)}-b^{l} \frac{\kappa \beta_{p}}{y^{p}} \frac{\partial y}{\partial y^{e}} & -\frac{1}{p_{b}} \tau_{w} \frac{y}{x}+\frac{\partial p_{b}}{\partial \omega}(\cdot)-b^{l} \frac{\partial i(\cdot)}{\partial \omega} \\
J_{3,1}^{(3)} & J_{3,2}^{(3)} & J_{3,3}^{(3)} & \beta_{y^{e}}\left(s_{c}-\tau_{w}\right) l^{d}+\left(\beta_{y^{e}}-y^{e}\right) \frac{\partial i(\cdot)}{\partial \omega} \\
0 & 0 & \omega \kappa\left(\kappa_{w}-1\right) \frac{\beta_{p}}{y_{p}} \frac{\partial y}{\partial y^{e}} & 0
\end{array}\right)
$$

where the $J_{(i, j)}^{(3)}$ are elements of the $3 \times 3$ subsystem (53). When $\beta_{p}=0$ the matrix becomes

$$
J^{(4)}(0)=\left(\begin{array}{ccc|c}
J_{1,1}^{(3)} & J_{1,2}^{(3)} & J_{1,3}^{(3)} & -m \frac{\partial i(\cdot)}{\partial \omega} \\
J_{2,1}^{(3)} & J_{2,2}^{(3)} & J_{2,3}^{(3)} & -\frac{1}{p_{b}} \tau_{w} \frac{y}{x}+\frac{\partial p_{b}}{\partial \omega}(\cdot)-b^{l} \frac{\partial i(\cdot)}{\partial \omega} \\
J_{3,1}^{(3)} & J_{3,2}^{(3)} & J_{3,3}^{(3)} & \beta_{y^{e}}\left(s_{c}-\tau_{w}\right) l^{d}+\left(\beta_{y^{e}}-y^{e}\right) \frac{\partial i(\cdot)}{\partial \omega} \\
\hline 0 & 0 & 0 & 0
\end{array}\right) .
$$

From the well known property of a square matrix

$$
A=\left(\begin{array}{cc}
A_{11} & 0 \\
D & A_{22}
\end{array}\right)
$$

where $A_{11}$ and $A_{22}$ are square matrices, that

$$
\operatorname{det} A=\left(\operatorname{det} A_{11}\right)\left(\operatorname{det} A_{22}\right)
$$

we can conclude that $\operatorname{det} J^{(4)}\left(\beta_{p}\right)=\left(\operatorname{det} J^{(3)}\right)\left(\operatorname{det} A_{22}\right)=0$. The determinant of the lower right $1 \times 1$ matrix $A_{22}$ is also the eigenvalue of the $1 \times 1$ matrix and equals 0 . Hence one eigenvalue of $J^{(4)}\left(\beta_{p}\right)$ is zero, which was obvious because $J^{(4)}\left(\beta_{p}\right)$ is singular. The determinant of $J^{(3)}$ is negative as we have seen from the proof of Proposition 2. From the same proof we can conclude that the eigenvalues of $J^{(3)}$ all have negative real parts. Hence we know that the eigenvalues of $J^{(4)}\left(\beta_{p}\right)$ consist of the three negative eigenvalues of $J^{(3)}$ and one zero eigenvalue.

Now we have to show that small values of $\beta_{p}$ lead to local asymptotic stability of the system. From continuity of the eigenvalues of $J^{(4)}\left(\beta_{p}\right)$ we see that sufficiently small but positive values of $\beta_{p}$ do not change the sign of the three eigenvalues that formerly had negative real parts. If we can show that the determinant of $J^{(4)}\left(\beta_{p}\right)$ is positive then we will have shown that the formerly zero eigenvalue takes negative values, because the product of four negative eigenvalues is positive. 
The determinant of $J^{(4)}\left(\beta_{p}\right)$ turns out to be

$$
\operatorname{det} J^{(4)}\left(\beta_{p}\right)=-\omega \kappa\left(\kappa_{w}-1\right) \frac{\beta_{p}}{y_{p}} \frac{\partial y}{\partial y^{e}} \beta_{y^{e}}\left(s_{c}-\tau_{w}\right) l^{d}\left|\begin{array}{cc}
-m \frac{\partial i(\cdot)}{\partial m} & -m \frac{\partial i(\cdot)}{\partial b^{l}} \\
\frac{\partial p_{b}}{\partial m}(\cdot)-\frac{\mu}{p_{b}} & \frac{\partial p_{b}}{\partial b^{l}}(\cdot)-\mu
\end{array}\right| .
$$

We know from the proof of asymptotic stability for the 3D system that the latter determinant is positive whenever the assumptions made in the proposition are met, because it equals the second order principal minor $\left|J_{M, 3}^{(3)}\right|$. Additionally we know that $s_{c}-\tau_{w}>0$ and $\kappa_{w}-1<0$. All other variables are positive, thus we can conclude that the $\operatorname{det} J^{(4)}\left(\beta_{p}\right)>0$.

\section{Proof of Proposition 4}

The Jacobian the dynamical system (55) is given by

$$
J^{(5)}=\left(\begin{array}{ccccc}
J_{1,1}^{(4)} & J_{1,2}^{(4)} & J_{1,3}^{(4)}-m \kappa \kappa_{p} \beta_{w} \frac{1}{x l} \frac{\partial y}{\partial y^{e}} & J_{1,4}^{(4)} & m \kappa \kappa_{p} \beta_{w} \frac{y}{x l^{2}} \\
J_{2,1}^{(4)} & J_{2,2}^{(4)} & J_{2,3}^{(4)}-b^{l} \kappa \kappa_{p} \beta_{w} \frac{1}{x l} \frac{\partial y}{\partial y^{e}} & J_{2,4}^{(4)} & b^{l} \kappa \kappa_{p} \beta_{w} \frac{y}{x l^{2}} \\
J_{3,1}^{(4)} & J_{3,2}^{(4)} & J_{3,3}^{(4)} & J_{3,4}^{(4)} & 0 \\
J_{4,1}^{(4)} & J_{4,2}^{(4)} & J_{4,3}^{(4)}+\frac{\omega \kappa\left(1-\kappa_{p}\right) \beta_{w}}{x l} \frac{\partial y}{\partial y^{e}} & J_{4,4}^{(4)} & -\omega \kappa\left(1-\kappa_{p}\right) \beta_{w} \frac{y}{x l^{2}} \\
-l i i_{q} \frac{\partial q}{\partial m} & -l i_{q} \frac{\partial q}{\partial b^{l}} & -l \frac{\partial i(\cdot)}{\partial y^{e}} & -l i_{q} \frac{\partial q}{\partial \omega} & 0
\end{array}\right)
$$

where the $J_{(i, j)}^{(4)}$ are the elements of $J^{(4)}$. The proof of the proposition follows the same route as the proof of Proposition 3. With $\beta_{w}=0$ the last column contains only zeros, thus the matrix is singular and must possess one zero eigenvalue and four eigenvalues with negative real parts.

Applying some row operations to the determinant which do not change its value at all we obtain the representation:

$$
J^{(5)}=(\operatorname{col} 1, \operatorname{col} 2, \operatorname{col} 3, \operatorname{col} 4, \operatorname{col} 5)
$$

when

$$
\begin{aligned}
\operatorname{col} 1= & \left(-m \frac{\partial i(\cdot)}{\partial m},-m \frac{\partial i(\cdot)}{\partial b^{l}},-m \frac{\partial i(\cdot)}{\partial y^{e}}-m \frac{\kappa \beta_{p}}{y^{p}} \frac{\partial y}{\partial y^{e}}-\frac{m \kappa \kappa_{p} \beta_{w}}{x l} \frac{\partial y}{\partial y^{e}}\right. \\
& \left.-m \frac{\partial i(\cdot)}{\partial \omega}, m \kappa \kappa_{p} \beta_{w} \frac{y}{x l^{2}}\right)^{\top} \\
\operatorname{col} 2= & \left.\left(\frac{\partial p_{b}}{\partial m}(\cdot)-\frac{\mu}{p_{b}}, \frac{\partial p_{b}}{\partial b^{l}}(\cdot)-\mu\right)^{\top},-\frac{\tau_{w} \omega}{p_{b} x} \frac{\partial y}{\partial y^{e}},-\frac{1}{p_{b}} \tau_{w} \frac{y}{x}+\frac{\partial p_{b}}{\partial \omega}(\cdot), 0\right)^{\top} \\
\operatorname{col} 3= & \left(\begin{array}{ll}
0,0, \beta_{y^{e}}\left(\frac{\partial c}{\partial y^{e}}-1\right), \beta_{y^{e}}\left(s_{c}-\tau_{w}\right) l^{d}, 0
\end{array}\right)^{\top}
\end{aligned}
$$




$$
\begin{aligned}
& \operatorname{col} 4=\left(0,0, \omega \kappa\left(\frac{\left(1-\kappa_{p}\right) \beta_{w}}{x l^{d}}+\frac{\left(\kappa_{w}-1\right) \beta_{p}}{y_{p}}\right) \frac{\partial y}{\partial y^{e}}, 0,-\omega \kappa\left(1-\kappa_{p}\right) \beta_{w} \frac{y}{x l^{2}}\right)^{\top} \\
& \operatorname{col} 5=\left(0,0,-l \frac{\kappa \beta_{p}}{y^{p}} \frac{\partial y}{\partial y^{e}}-l \kappa \kappa_{p} \beta_{w} \frac{1}{x l} \frac{\partial y}{\partial y^{e}}, 0,-l \kappa \kappa_{p} \beta_{w} \frac{y}{x l^{2}}\right)^{\top}
\end{aligned}
$$

from which we can see that the sign of the determinant equals the determinant of the lower right-hand $3 \times 3$ system, because the upper $2 \times 2$ system has a positive determinant. The determinants of the lower right $3 \times 3$ sub matrix can be expressed as

$$
-\beta_{y^{e}}\left(s_{c}-\tau_{w}\right) l^{d}\left|\begin{array}{cc}
\omega \kappa\left(\left(\kappa_{w}-1\right)-\frac{1-\kappa_{p}}{\kappa_{p}}\right) \frac{\beta_{p}}{y_{p}} \frac{\partial y}{\partial y^{e}} & 0 \\
-l \frac{\kappa \beta_{p}}{y^{p}} \frac{\partial y}{\partial y^{e}}-l \kappa \kappa_{p} \beta_{w} \frac{1}{x l} \frac{\partial y}{\partial y^{e}} & -l \kappa \kappa_{p} \beta_{w} \frac{y}{x l^{2}}
\end{array}\right|
$$

from which we can conclude that the determinant $\left|J^{(5)}\right|$ is negative.

Steady state or trend values are indicated by a sub- or superscript ' $o$ '. When no confusion arises, letters $F, G, H$ may also define certain functional expressions in a specific context. A dot over a variable $x=x(t)$ denotes the time derivative, a caret its growth rate; $\dot{x}=d x / d t, \hat{x}=\dot{x} / x$. In the numerical simulations, flow variables are measured at annual rates.

As far as possible, the notation tries to follow the logic of using capital letters for level variables and lower case letters for variables in intensive form, or for constant (steady state) ratios. Greek letters are most often constant coefficients in behavioural equations (with, however, the notable exceptions being $\pi, \omega$ ).

\section{References}

Abreu, D. and Brunnermeier, M. (2003), 'Bubbles and crashes', Econometrica 71, 173-204.

Asada, T., Chiarella, C., Flaschel, P., Mouakil, T., Proaño, C. and Semmler, W. (2010), 'Stabilizing an unstable economy: On the choice of proper policy measures', Economics: The Open-Access, Open-Assessment E-Journal 4(2010-21).

Asada, T., Chiarella, C., Flaschel, P., Mouakil, T., Proaño, C. and Semmler, W. (2011), 'Stock-flow interactions, disequilibrium macroeconomics and the role of economic policy', Journal of Economic Surveys . Forthcoming.

Baumol, W. J. (1952), 'The transactions demand for cash: An inventory theoretic approach', The Quarterly Journal of Economics 66(4), 545-556.

Chiarella, C., Dieci, R. and He, X. (2009), Heterogeneity, market mechanisms, and asset price dynamics, in T. Hens and K. R. Schenk-Hoppé, eds, 'Handbook of Financial Markets: Dynamics and Evolution', North-Holland, pp. 277-344. 
Chiarella, C., Flaschel, P. and Franke, R. (2005), Foundations for a Disequilibrium Theory of the Business Cycle: Qualitative Analysis and Quantitative Assessment, Cambridge University Press.

Chiarella, C., Flaschel, P., Franke, R. and Semmler, W. (2006), A high-dimensional model of real-financial market interaction: The cascade of stable matrices approach, in C. Chiarella, R. Franke, P. Flaschel and W. Semmler, eds, 'Quantitative and Empirical Analysis of Nonlinear Dynamic Macromodels', Vol. 277 of Contribution to Economic Analysis, Elsevier, chapter 11, pp. 359-384.

Franke, R. and Semmler, W. (1999), 'Bond rate, loan rate and tobins $q$ in a temporary equilibrium model of the financial sector', Metroeconomica 50(3), 351385.

Köper, C. (2003), Real-Financial Interaction in Contemporary Models of AS-AD Growth, Peter Lang, Bern.

Minsky, H. P. (1982), Stabilizing an Unstable Economy, 2 edn, McGraw Hill, New York.

Palley, T. I. (2011), Quantitative easing: A Keynesian critique. Political Economy Research Institute, University of Massachusetts, Amherst, Working Paper Series No. 252.

Rødseth, A. (2000), Open Economy Macroeconomics, Cambridge University Press, Cambridge, UK.

Sargent, T. J. (1987), Macroeconomic Theory, 2nd edn, Academic Press, New York.

Tobin, J. (1969), 'A general equilibrium approach to monetary theory', Journal of Money, Credit, and Banking 1(1), 15-29.

Tobin, J. (1980), Asset Accumulation and Economic Activity, Blackwell, Oxford, Basil.

Tobin, J. (1982), 'Money and the macroeconomic process', Journal of Money, Credit and Banking 14, 171-204. 